\title{
EQUIVALENCE OF A TANGLE CATEGORY AND A CATEGORY OF INFINITE DIMENSIONAL $\mathrm{U}_{q}\left(\mathfrak{s l}_{2}\right)$-MODULES
}

\author{
K. IOHARA, G. I. LEHRER, AND R. B. ZHANG
}

\begin{abstract}
It is very well known that if $V$ is the simple 2-dimensional representation of $\mathrm{U}_{q}\left(\mathfrak{s l}_{2}\right)$, the category of representations $V^{\otimes r}, r=0,1,2, \ldots$, is equivalent to the Temperley-Lieb category $\mathrm{TL}(q)$. Such categorical equivalences between tangle categories and categories of representations are rare. In this work we give a family of new equivalences by extending the above equivalence to one between the category of representations $M \otimes V^{\otimes r}$, where $M$ is a projective Verma module of $\mathrm{U}_{q}\left(\mathfrak{s l}_{2}\right)$ and the type $B$ Temperley-Lieb category $\mathbb{T} \mathbb{B}(q, Q)$, realised as a subquotient of the tangle category of Freyd, Yetter, Reshetikhin, Turaev and others.
\end{abstract}

\section{Contents}

1. Introduction

2. The Temperley-Lieb category of type $B$

3. Multipolar and affine Temperley-Lieb categories

4. Quantum Schur-Weyl duality

\section{INTRODUCTION}

Suppose that $\mathbb{T}$ is a subquotient of the tangle category of Freyd-Yetter [11] and Reshitikhin-Turaev 24] (see below). There are many examples (cf. [24,25]) of a functor $\mathcal{F}: \mathbb{T} \longrightarrow \operatorname{Rep}\left(\mathrm{U}_{q}\right)$, where $\operatorname{Rep}\left(\mathrm{U}_{q}\right)$ is a full subcategory of the category of representations of a quantum group $\mathrm{U}_{q}$. However it is rare that $\mathcal{F}$ is an equivalence. One such case is when $\mathbb{T}=\mathrm{TL}(q)$, the Temperley-Lieb category. It is well known (see, e.g., 44,10, 17, 19, 24, 25) that the Temperley-Lieb category is isomorphic to the full subcategory of $\mathrm{U}_{q}\left(\mathfrak{s l}_{2}\right)$-modules with objects $V^{\otimes r}$ for $r=0,1, \ldots$, where $V$ is the 2-dimensional simple module. This isomorphism underlies the quantum group theoretical construction of the celebrated Jones polynomial of knots [17. In this work we show that this isomorphism of categories extends to a family of equivalences between the Temperley-Lieb category of type $B$, and a certain category of infinite dimensional representations of $\mathrm{U}_{q}\left(\mathfrak{s l}_{2}\right)$.

Specifically, we generalise the classical isomorphism of categories above to an isomorphism of categories between the Temperley-Lieb category $\mathbb{\mathbb { R }} \mathbb{B}(q, Q)$ of type $B$ [14] with appropriate parameters and the full subcategory $\mathcal{T}$ of $\mathrm{U}_{q}\left(\mathfrak{s l}_{2}\right)$-modules generated by the objects $M \otimes V^{\otimes r}(r=0,1, \ldots)$ where $M$ is a fixed projective Verma module. The choice of parameter $Q$ depends on $M$.

Received by the editors November 20, 2019, and, in revised form, November 25, 2020.

2020 Mathematics Subject Classification. Primary 17B37, 20G42; Secondary 81R50.

Key words and phrases. Tangle category, Temperley-Lieb category of type $B$, Verma module. Partially supported by the Australian Research Council. 
A consequence of the isomorphism between the categories $\mathbb{T} \mathbb{B}(q, Q)$ and $\mathcal{T}$ is a generalised version of Schur-Weyl duality, identifying the endomorphism algebra $\operatorname{End}_{\mathcal{T}}\left(M \otimes V^{\otimes r}\right)$ with the Temperley-Lieb algebra $\mathbb{\mathbb { L }} \mathbb{B}_{r}(q, Q)$ of type $B_{r}$, where $\mathbb{\square} \mathbb{B} \mathbb{B}_{r}(q, Q)$ is the endomorphism algebra of an object of the category $\mathbb{T} \mathbb{B}(q, Q)$ in analogy with [20. This enables us to bring into play the cellular structure of the Temperley-Lieb algebra, which we use to determine a precise criterion for the semisimplicity of the endomorphism algebra $\operatorname{End}_{\mathcal{T}}\left(M \otimes V^{\otimes r}\right)$.

The proofs of the main results rely on a new description of the TemperleyLieb category $\mathbb{T} \mathbb{B}(q, Q)$ of type $B$ as a subquotient $\mathcal{T} \mathcal{L B}(q, \Omega)$ of the coloured framed tangle category extensively studied by Freyd, Yetter [11, Turaev, Reshitikhin $[24,25,27]$ and others. The category $\mathbb{T} \mathbb{R}(q, Q)$ was defined in [14] as a category of marked diagrams (i.e., "blob diagrams"), and the new category $\mathcal{T} \mathcal{L B}(q, \Omega)$ is described in terms of polar tangle diagrams. Ostensibly the two categories are very different, but nevertheless we are able to construct in Theorem 3.27an explicit isomorphism between them with appropriate parameter matchings.

Two key ideas are involved in the reformulation of $\mathbb{\mathbb { R }} \mathbb{B}(q, Q)$. One is the uniform description of the affine Hecke algebra, affine Temperley-Lieb algebra and other related algebras given in [14, where the unifying object is the braid group of type $B$. The affine Temperley-Lieb algebra is a quotient of the group algebra of this group, which factors through the affine Hecke algebra, while the Temperley-Lieb algebra of type $B$ is in turn a quotient of the affine Temperley-Lieb algebra. The other is the embedding of the braid group of type $B_{r}$ into the braid group of type $A_{r+1}$ (see Remark 3.2), which leads to a description of the group algebra of the former and its quotient algebras in terms of polar tangle diagrams [1,8. Our construction of $\mathcal{T} \mathcal{L B}(q, \Omega)$ is a categorical generalisation of this.

We first introduce in Section 3.1 a category $\mathcal{R} \mathcal{T}$ of coloured unoriented tangle diagrams up to regular isotopy [11,24,27]. The objects of the category are sequences of elements of $\mathcal{C}:=\{m, v\}$, and the modules of morphisms are spanned by a class of coloured un-oriented tangle diagrams up to regular isotopy. This category has many interesting quotient categories closely related to categories of representations of quantum groups $[2,26,28$.

By imposing Temperley-Lieb skein relations (3.3), (3.4), (as well as their consequence which we call free loop removal (3.5) to morphisms of $\mathcal{R} \mathcal{T}$, we obtain a quotient category $\mathcal{M T} \mathcal{L}(q)$, the multipolar Temperley-Lieb category. The oneparameter multi-polar Temperley-Lieb category $\mathcal{M T} \mathcal{L}(q, \Omega)$ is obtained as a quotient of $\mathcal{M T} \mathcal{L}(q)$ by specialising the two central morphisms given by (3.7) to appropriate scalars related to $\Omega$ as described in (3.9). The second version $\mathcal{T} \mathcal{L B}(q, \Omega)$ of the Temperley-Lieb category of type $B$ is the full subcategory of $\mathcal{M T} \mathcal{L}(q, \Omega)$ generated by objects of the form $\left(m, v^{r}\right):=(m, \underbrace{v, \ldots, v}_{r})$ for all $r \in \mathbb{Z}_{\geqslant 0}$. The category $\mathcal{M T} \mathcal{L}(q, \Omega)$ contains the finite Temperley-Lieb category $\mathcal{T} \mathcal{L}(q)$ as a full subcategory in two different ways and in one of these it is also contained in $\mathcal{T} \mathcal{L B}(q, \Omega)$. There exists a tensor functor $\mathcal{T} \mathcal{L B}(q, \Omega) \times \mathcal{T} \mathcal{L}(q) \longrightarrow \mathcal{T} \mathcal{L} \mathcal{B}(q, \Omega)$.

The structure of the category $\mathcal{T} \mathcal{L B}(q, \Omega)$ is studied in depth in Section 3.4, and that of $\mathbb{T} \mathbb{B}(q, Q)$ is summarised in Section 2 . An explicit isomorphism between $\mathcal{T} \mathcal{L B}(q, \Omega)$ and $\mathbb{T} \mathbb{R}(q, Q)$ is constructed in Theorem 3.27 . 
We construct in Theorem 4.5 a tensor functor $\hat{\mathcal{F}}$ from the category $\mathcal{R} \mathcal{T}$ to the category $\mathcal{O}_{\text {int }}$ of $\mathrm{U}_{q}\left(\mathfrak{s l}_{2}\right)$-modules. This functor is shown to factor through the multipolar Temperley-Lieb category $\mathcal{M T} \mathcal{L}(q, \Omega)$ with parameter $\Omega$, whose dependence on $M$ is given by (4.18). This induces a functor from $\mathcal{M T} \mathcal{L}(q, \Omega)$ to $\mathcal{O}_{\text {int }}$, which restricts to a functor $\mathcal{F}^{\prime}: \mathcal{T} \mathcal{L B}(q, \Omega) \longrightarrow \mathcal{T}$, where $\mathcal{T}$ is the full subcategory of $\mathcal{O}_{\text {int }}$ mentioned above. We are able to show in Theorem 4.9 that $\mathcal{F}^{\prime}$ is an isomorphism of categories. Our proof of this theorem uses the fact that our category equivalence extends the well known one between the Temperley-Lieb category and the category with objects the tensor powers of the 2-dimensional simple $\mathrm{U}_{q}\left(\mathfrak{s l}_{2}\right)$-module (see, e.g., 4, 10, 19]), which we refer to as the "classical case".

We note that this latter equivalence leads to analogous statements when $q$ is a root of unity in the classical case (see [2]), and we expect this to be the case in our more general situation.

The endomorphism algebra $\operatorname{TLB}_{r}(q, \Omega)$ of the object $\left(m, v^{r}\right)$ in $\mathcal{T} \mathcal{L B}(q)$ and the Temperley-Lieb algebra $\mathbb{\mathbb { L }} \mathbb{B}_{r}(q, Q)$ of type $B_{r}$ are isomorphic for appropriate parameters $Q$ and $\Omega$. The above isomorphism of categories implies that as associative algebra $\operatorname{End}_{\mathcal{T}}\left(M \otimes V^{\otimes r}\right)$ is isomorphic to $\operatorname{TLB}_{r}(q, \Omega)$, and hence $\mathbb{T} \mathbb{R} \mathbb{B}_{r}(q, Q)$, for all $r \geqslant 0$, which is a generalisation of the well-known classical Schur-Weyl duality [4, 10, 19] to the present case involving Verma modules.

Various new categories arise naturally from our constructions, which are interesting in their own right. These could lead to further connections between the (generally infinite dimensional) endomorphism algebras in these categories, and certain subcategories of the representation category of $\mathrm{U}_{q}\left(\mathfrak{s l}_{2}\right)$. In particular, even in the affine case, an equivalence would be interesting (cf. [6]).

We note finally that other work has been done in the general context of SchurWeyl duality for modules related to those in this work; see e.g., 6, 9, 16].

Throughout the paper, we work over the ground field $\mathcal{K}_{0}:=\mathbb{C}\left(q^{\frac{1}{k}}\right)$, where $q$ is an indeterminate over $\mathbb{C}$ such that $\left(q^{\frac{1}{k}}\right)^{k}=q$ for some fixed positive integer $k$. For any invertible scalar $t \in \mathcal{K}_{0}$ we write $\delta_{t}:=-\left(t+t^{-1}\right)$.

\section{The Temperley-Lieb CAtegory of type $B$}

2.1. The Temperley-Lieb category of type $B$. Let $R$ be a ring and let $q, Q \in R$ be invertible elements. We begin by recalling the definition of the Temperley-Lieb category $\mathbb{\mathbb { R }} \mathbb{B}(q, Q)$ of type $B$ from $[14$ ( see also $[7,23])$. The objects of $\mathbb{\llbracket} \mathbb{B}(q, Q)$ are the integers $t \in \mathbb{Z}_{\geqslant}$. A $(\mathbb{T} \mathbb{B})$ diagram $D: t \longrightarrow s$ is a "marked Temperley-Lieb diagram" from $t$ to $s$, and $\operatorname{Hom}_{\mathbb{T} \mathbb{B}(q, Q)}(t, s)$ is the free $R$ module with basis the set of $\mathbb{T} \mathbb{B}$ diagrams: $t \longrightarrow s$.

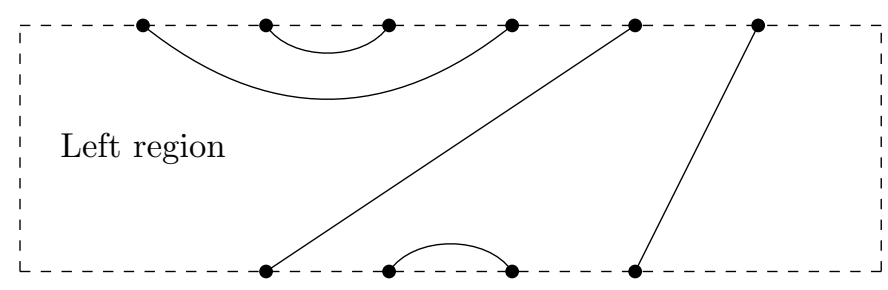

FiguRE 1 
To define marked diagrams, recall that a TL diagram $t \longrightarrow s$ divides the "fundamental rectangle" into regions, of which there is a unique leftmost one (see Figure1, which is a TL diagram: $4 \longrightarrow 6$ ). A marked diagram is a TL diagram in which the boundary arcs of the left region may be marked with dots. Thus the TL diagram depicted in Figure 1 has 2 markable arcs, and a corresponding marked diagram is shown in Figure 2

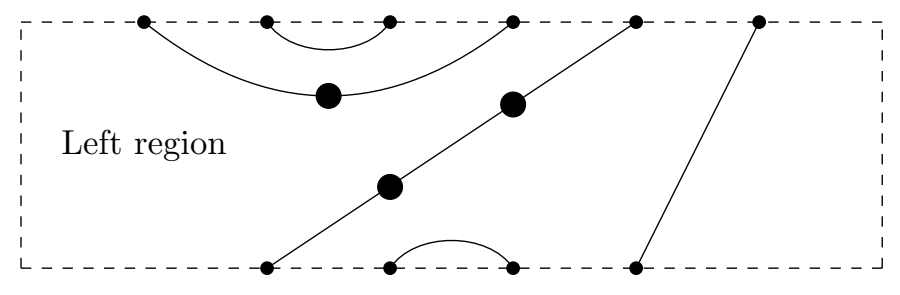

FigURE 2

These marked diagrams are composed via concatenation, just as TL-diagrams, with three rules to bring them to "standard form", which is a diagram with at most one mark on each eligible arc. The rules are (recalling that for any invertible element $\left.x \in R, \delta_{x}=-\left(x+x^{-1}\right)\right)$ :

(i) If $D$ is a (marked) diagram and $L$ is a loop with no marks then $D \amalg L=\delta_{q} D$.

(ii) If, in (i), $L$ is a loop with 1 mark then $D \amalg L=\left(\frac{q}{Q}+\frac{Q}{q}\right) D$.

(iii) If an arc or loop of a diagram $D$ has more than 1 mark and $D^{\prime}$ is obtained from $D$ by removing one mark from that arc or loop, then $D=\delta_{Q} D^{\prime}$.

Definition 2.1. The category $\mathbb{T} \mathbb{B}(q, Q)$ has objects $\mathbb{Z}_{\geqslant 0}$ and morphisms which are $R$-linear combinations of marked diagrams, subject to the rules (2.1).

It is evident that $\operatorname{Hom}_{\mathbb{U} \mathbb{B}(q, Q)}(t, s)$ has basis consisting of TLB (marked) diagrams with at most one mark on each boundary arc of the left region. We shall refer to these as "marked diagrams"; the next result counts them.

Proposition 2.2. For integers $t, k \geqslant 0$, the number $b(t, t+2 k)$ of marked diagrams $t \longrightarrow t+2 k$ depends only on $t+k$. If $t+k=m$, then the number of these is $d(m)=\left(\begin{array}{c}2 m \\ m\end{array}\right)$.

Proof. Given a marked diagram $t \longrightarrow t+2 k$, one obtains a diagram $0 \longrightarrow 2(t+k)$ by rotating the bottom of the diagram through $180^{\circ}$ until it becomes part of the top, pulling all the relevant arcs appropriately. This is illustrated in Figure 3 for the diagram in Figure 2 (but note that we are applying this construction only to standard diagrams, which have at most one mark on each arc).

This shows that $b(t, t+2 k)=b(0,2(t+k)$, which proves the first statement. Write $d(m)=b(0,2 m)$, and following [15, §4.3], we write $d(x)=\sum_{i=0}^{\infty} d(i) x^{i}$, where $d(0)=1$. Now if the $2 m$ upper dots are numbered $1,2, \ldots, 2 m$ from left to right any marked diagram $D: 0 \longrightarrow 2 m$ will join 1 to an even numbered dot, say $2 i$. For 

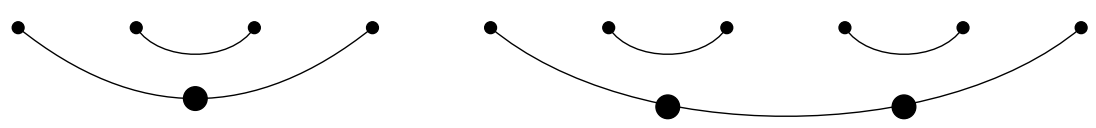

FiguRE 3

fixed $i$, the number of such $D$ is $2 c(i-1) d(m-i)$, where $c(i)$ is the Catalan number (cf. [15]) (since the arc $(1,2 i)$ may be either marked or unmarked). It follows that

$$
d(m)=2 \sum_{i=1}^{m} c(i-1) d(m-i) .
$$

Multiplying (2.2) by $x^{m}$ and summing, we obtain

$$
d(x)=\frac{1}{1-2 x c(x)},
$$

where $c(x)=\sum_{i=0}^{\infty} c(i) x^{i}=\sum_{i=0}^{\infty} \frac{1}{i+1}\left(\begin{array}{c}2 i \\ i\end{array}\right) x^{i}$. Now from the above relation, one sees easily that

$$
\frac{\partial}{\partial x}(x c(x))=x c^{\prime}(x)+c(x)=\sum_{n=0}^{\infty}\left(\begin{array}{c}
2 n \\
n
\end{array}\right) x^{n} .
$$

Now differentiating the relation $x c(x)^{2}=c(x)-1$, we obtain

$$
(1-2 x c(x))^{-1}=d(x)=\frac{c^{\prime}(x)}{c(x)^{2}}=\frac{\partial}{\partial x}(x c(x)-1),
$$

and the result follows from (2.4).

2.2. Generators and cellular structure. The usual Temperley-Lieb category $\mathcal{T} \mathcal{L}(q)$ (see Definition 3.5) is a subcategory of $\mathbb{T} \llbracket \mathbb{B}(q, Q)$, with the same objects, and morphisms which are $R$-linear combinations of unmarked diagrams. Thus there is a faithful functor

$$
\mathcal{T} \mathcal{L}(q) \longrightarrow \mathbb{T} \mathbb{B}(q, Q)
$$

as well as a "tensor product" functor

$$
\mathbb{T} \mathbb{B}(q, Q) \times \mathcal{T} \mathcal{L}(q) \longrightarrow \mathbb{T} \mathbb{B}(q, Q),
$$

given by juxtaposing diagrams. Note that the functor (2.5) restricts to the usual tensor product on $\mathcal{T} \mathcal{L}(q)$.

The category $\mathcal{T} \mathcal{L}(q)$ is generated, under composition and tensor product by the morphisms $A, U, I$ depicted in Figure 4 subject to the obvious relations.

The category $\mathbb{T} \mathbb{B}(q, Q)$ is generated by the generators $A, U, I$ of $\mathcal{T} \mathcal{L}$, with $C_{0}$ added as shown. Evidently it follows from (2.1)(iii) that $C_{0}$ satisfies $C_{0}^{2}=\delta_{Q} C_{0}$ and from (2.1) (ii) that $A\left(C_{0} \otimes I\right) U=\frac{q}{Q}+\frac{Q}{q}$. For $n=1,2, \ldots$, the algebra $\operatorname{Hom}_{\mathbb{T} \mathbb{B}(q, Q)}(n, n)$ is the Temperley-Lieb algebra $\mathbb{T} \llbracket \mathbb{B}_{n}(q, Q)$ of type $B_{n}$. It has a cellular structure described as follows.

Given $n \in \mathbb{Z}_{>0}$, define $\Lambda_{B}(n)=\{t \in \mathbb{Z}|| t \mid \leqslant n$ and $t \equiv n(\bmod 2)\}$. For $t \in \Lambda_{B}(n)$ define $M(t)$ as the set of monic diagrams $D:|t| \longrightarrow n$ in which no through string is marked. Here "monic" means that the diagram has a left inverse in the category $\mathbb{T} \mathbb{B}(q, Q)$, which is equivalent to the requirement that all arcs with 


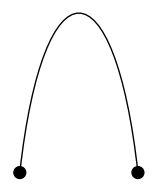

A

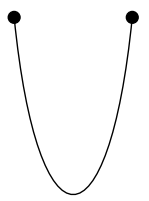

$\mathrm{U}$

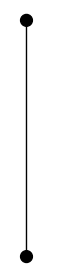

I

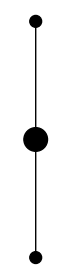

$C_{0}$

FiguRE 4

a lower boundary point also have an upper boundary point. For each $t \in \Lambda_{B}(n)$, there is an injective map $\beta_{t}: M(t) \times M(t) \longrightarrow \mathbb{T} \mathbb{\mathbb { B } _ { n }}(q, Q)$ given by

$$
\beta_{t}\left(D_{1}, D_{2}\right)=\left\{\begin{array}{l}
D_{2} D_{1}^{*} \text { if } t \geqslant 0 \\
D_{2}\left(C_{0} \otimes I^{\otimes(|t|-1)}\right) D_{1}^{*} \text { if } t<0
\end{array}\right.
$$

where $D^{*}$ is the diagram obtained from $D$ by reflection in a horizontal.

The next result is straightforward.

Proposition 2.3. Maintain the above notation.

(1) Let $C:=\amalg_{t \in \Lambda_{B}(n)} \beta_{t}: \amalg_{t \in \Lambda_{B}(n)} M(t) \times M(t) \longrightarrow \mathbb{T} \llbracket \mathbb{B}_{n}(q, Q)$. The image of $C$ is a basis of $\mathbb{T} \mathbb{B} \mathbb{B}_{n}(q, Q)$. Write $\beta_{t}(S, T)=C_{S, T}^{t}$ for the basis elements $(S, T \in M(t))$.

(2) The basis $C_{S, T}^{t}\left(t \in \Lambda_{B}(n), S, T \in M(t)\right)$ is a cellular basis of $\mathbb{\mathbb { Q }} \mathbb{B}_{n}(q, Q)$.

Remark 2.4. An analogous result for Temperley-Lieb algebras of type $D$ is proved in [21.

2.3. Cell modules for $\mathbb{T} \llbracket \mathbb{B}_{n}(q, Q)$. We give a description of the cell modules corresponding to the cellular structure given in Proposition 2.3 and compute their dimension, although this is implicit in the results of [14]. The cell module $W_{t}(n)$ $(t \in \Lambda(n))$ has basis the set $M(t)$, with $\mathbb{\mathbb { R }} \mathbb{B}_{n}(q, Q)$-action defined in the usual way by multiplication of diagrams.

Let $u(t, k)=\operatorname{rank}\left(W_{t}(|t|+2 k)\right)$. We have seen (Proposition 2.2) that $u(0, k)=$ $\left(\begin{array}{c}2 k \\ k\end{array}\right)$. This is the case $t=0$ of the following result.

Proposition 2.5. We have

$$
\operatorname{dim} W_{t}(|t|+2 k)=u(t, k)=\left(\begin{array}{c}
|t|+2 k \\
k
\end{array}\right) .
$$

Proof. It clearly suffices to consider the case $t \geqslant 0$. We prove the result by induction on the pair $t, k$, the result being known for $t=0$ (Proposition 2.2), while for $k=0$, clearly $u(t, 0)=1=\left(\begin{array}{c}|t| \\ 0\end{array}\right)$.

Now the same argument as in [15, Prop. 5.2], involving rotation of the bottom row of a monic diagram $|t| \longrightarrow|t|+2 k$ through $180^{\circ}$ to obtain a diagram $0 \longrightarrow$ $2|t|+2 k$, shows that we have the following recursion for $u(t, k)$. Assume $t, k \geqslant 1$. Then

$$
u(t, k)=u(t-1, k)+u(t+1, k-1) .
$$

Hence by induction, $u(t, k)=\left(\begin{array}{c}t-1+2 k \\ k\end{array}\right)+\left(\begin{array}{c}t+1+2(k-1) \\ k-1\end{array}\right)=\left(\begin{array}{c}t+2 k \\ k\end{array}\right)$. 


\section{Multipolar and affine Temperley-Lieb Categories}

Certain categories of framed tangles, both oriented and unoriented [11, and of ribbon graphs [24], have played important roles in the construction of topological invariants of link and 3-manifold. We now develop an alternative formulation of the Temperley-Lieb category $\mathbb{T} \llbracket \mathbb{B}(q, Q)$ of type $B$ in terms of framed tangles.

3.1. A subcategory of tangle diagrams. We introduce here a category of unoriented tangles up to regular isotopy in the sense of [11, which we denote by $\mathcal{R T}$. Our category is a subcategory of the category $S-\mathbb{R} \mathbb{T}$ ang, where $S$ is the set $\mathcal{C}:=\{m, v\}$ which is defined in [11, Def. 3.1]. We shall use the language of tangle diagrams, although an equivalent formulation could use the language of coloured ribbon graphs.

The objects of $\mathcal{R} \mathcal{T}$ are sequences of elements of $\mathcal{C}=\{m, v\}$, which are called "colours", where the empty sequence is allowed. The morphisms are spanned, over a base ring $R$, by unoriented tangles up to regular isotopy in the terminology of [11], coloured by $\mathcal{C}$. Such tangles are represented in "tangle diagrams" as unions of arcs; we say that an arc is horizontal if its end points are either both at the top or at the bottom of the tangle. An arc is vertical if it has end points and is not horizontal. To define (the subcategory) $\mathcal{R} \mathcal{T}$, we impose the following two conditions on morphisms.

(1) Closed arcs (i.e. those with no end points) are all coloured by $v$.

(2) Any arc coloured by $m \in \mathcal{C}$ is vertical, and no two such arcs cross.

The composition of morphisms is explained in [11] and is essentially by concatenation of tangle diagrams.

We call $\mathcal{R} \mathcal{T}$ the restricted coloured tangle category.

Since the two ends of any arc have the same colour, we will write $m$ or $v$ beside an arc to indicate its colour. The term "tangle diagram" will be abbreviated to "diagram" below.

Theorem 3.1. The category $\mathcal{R} \mathcal{T}$ has the following properties.

(1) There is a bi-functor $\otimes: \mathcal{R T} \times \mathcal{R} \mathcal{T} \longrightarrow \mathcal{R} \mathcal{T}$, called the tensor product, which is defined as follows. For any pair of objects $A=\left(a_{1}, \ldots, a_{r}\right)$ and $B=\left(b_{1}, \ldots, b_{s}\right)$, we have $A \otimes B=(A, B):=\left(a_{1}, \ldots, a_{r}, b_{1}, \ldots, b_{s}\right)$. The tensor product is bilinear on morphisms. Given diagrams $D$ and $D^{\prime}, D \otimes D^{\prime}$ is their juxtaposition with $D$ on the left.

(2) The morphisms are generated by the following elementary diagrams under tensor product and composition,
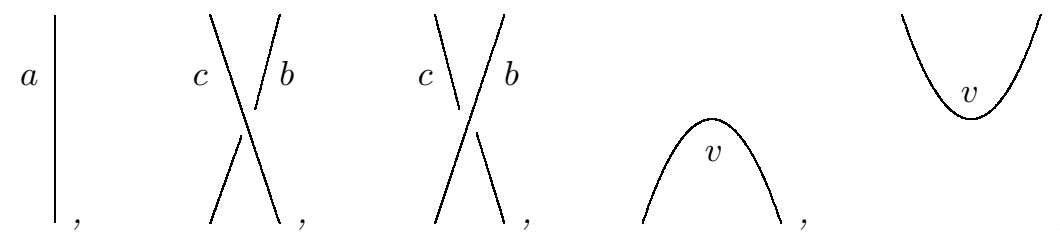

where $a, b, c \in \mathcal{C}=\{m, v\}$ with $b \neq c$ or $b=c=v$. 
(3) The defining relations among the above generators are as follows.

(a) Over and under crossings are inverses of each other: for all $a, b$ such that either $a \neq b$ or $a=b=v$,

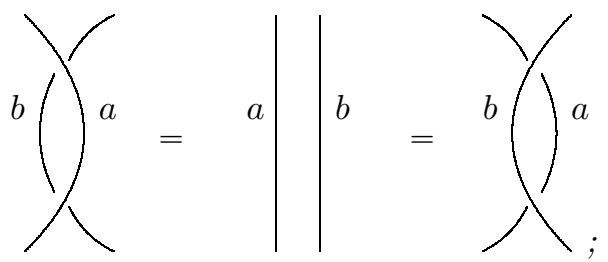

(b) Braid relation: for all $a, b, c$ at most one of which is equal to $m$,

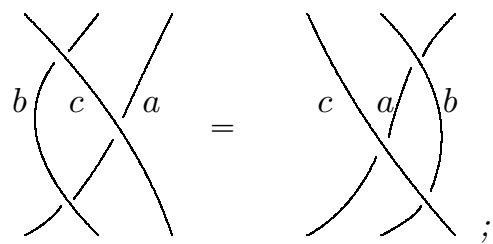

(c) Straightening relations:

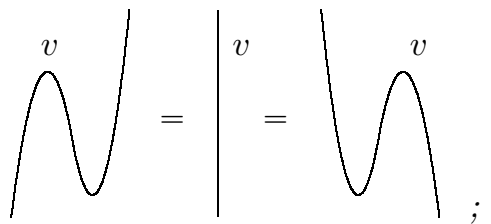

(d) Sliding relations: for all $a \in \mathcal{C}$,
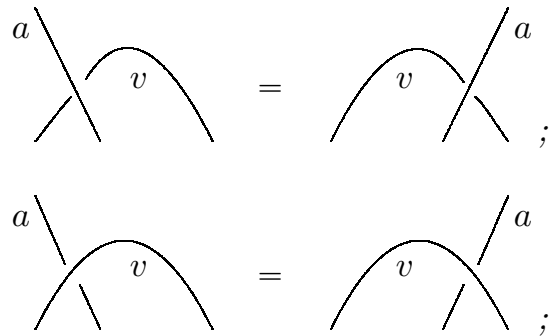

(e) Twists,

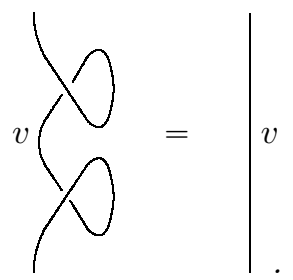

Proof. This was proved in [11, Theorem 3.5] and in [24, Lemma 5.3]. The result of [11, Theorem 3.5] in fact applies to several categories of tangles; the case which covers our theorem is that of $S-\mathbb{R} \mathbb{T}$ ang with $S=\mathcal{C}$. Note that [11, Theorem 3.5] 
does not involve colours, but this is not an issue as colours merely label components of tangles. We can also extract our theorem from [24, Lemma 5.3] by removing directions of ribbon graphs and forbidding coupons. There is also a direct proof along the lines of [20, Appendix].

An alternative representation. A second way of representing the category $\mathcal{R} \mathcal{T}$ is as follows. We depict arcs coloured by $m$ as thick arcs called poles, and arcs coloured by $v$ as thin arcs. This way a diagram automatically carries the information about the colours of its arcs, so that we may drop the letters for colours from the diagram. For example, we have the following diagram $\left(m, v^{2}, m, v^{2}\right) \rightarrow\left(v, m, v, m, v^{2}\right)$.

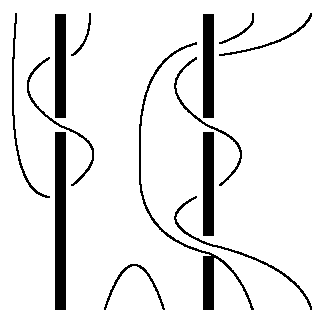

3.2. Some full subcategrories of $\mathcal{R} \mathcal{T}$. We denote by $\mathcal{B} \mathcal{T}$ the full subcategory of $\mathcal{R} \mathcal{T}$ with objects $\left(m, v^{r}\right)=(m, \underbrace{v, \ldots, v}_{r}), r=0,1,2, \ldots$ This is the category of "tangles of type $B$ ", or tangles with a pole.

We note that the automorphism group of the object $\left(m, v^{r}\right)$ in $\mathcal{B} \mathcal{T}$ (or $\mathcal{R} \mathcal{T}$ ) contains the Artin braid group $\Gamma_{r}$ of type $B_{r}$ (cf. [14, §2]). More generally, for any object $A \in \mathcal{C}^{N}$ in $\mathcal{R} \mathcal{T}$, the set $\Gamma(A)$ of diagrams $A \rightarrow A$ with only vertical arcs (i.e. arcs which are monotonic from top to bottom) forms a group. In particular $\Gamma\left(v^{r}\right)$ is the Artin braid group of type $A_{r}$, and $\Gamma\left(m, v^{r}\right) \cong \Gamma_{r}$ is the Artin braid group of type $B_{r}$ (cf. [14, §2]). It has generators $\xi_{1}, \sigma_{1}, \sigma_{2}, \ldots, \sigma_{r-1}$, and we shall freely use the notation in loc. cit.; in particular the generators $\xi_{1}, \sigma_{1}, \sigma_{2}, \ldots, \sigma_{r-1}$ satisfy the braid relations of type $B$ :

$$
\begin{aligned}
& \xi_{1} \sigma_{1} \xi_{1} \sigma_{1}=\sigma_{1} \xi_{1} \sigma_{1} \xi_{1} \\
& \xi_{1} \sigma_{i}=\sigma_{i} \xi_{1} \quad \text { if } i>1 \\
& \sigma_{i} \sigma_{j}=\sigma_{j} \sigma_{i} \quad \text { if }|i-j|>1 \\
& \sigma_{i} \sigma_{i+1} \sigma_{i}=\sigma_{i+1} \sigma_{i} \sigma_{i+1} \quad \text { for } i=1, \ldots, r-2 .
\end{aligned}
$$

These generators are identified with tangles as depicted in Figure 5. It is an enjoyable exercise to verify the first relation in (3.1) diagrammatically.

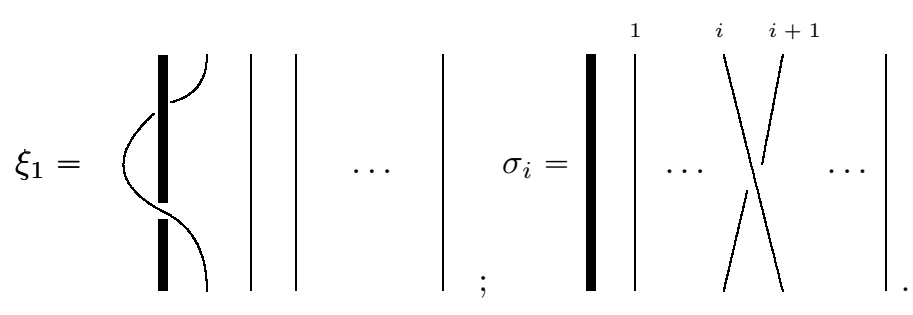

FiguRE 5. Generators $\xi_{1}$ and $\sigma_{i}$ 
The usual tangle category $\mathcal{T}_{0}$ is the full subcategory of $\mathcal{R} \mathcal{T}$ with objects $v^{r}$, $r=0,1,2, \ldots$ The tensor functor of $\mathcal{R} \mathcal{T}$ given by juxtaposition of tangles restricts to a tensor functor

$$
\mathcal{B T} \times \mathcal{T}_{0} \longrightarrow \mathcal{B} \mathcal{T}
$$

which will play an important role in this work.

Remark 3.2. When convenient, $\mathcal{T}_{0}$ will be identified with the subcategory of $\mathcal{B} \mathcal{T}$ whose morphisms have no entanglement with the pole in the obvious way.

3.3. Some subquotient categories of $\mathcal{R} \mathcal{T}$. There are various interesting quotient categories of $\mathcal{R} \mathcal{T}$, the endomorphisms of which include well-known algebras such as the affine Brauer algebra and affine Temperley-Lieb algebra. We define some quotient categories of $\mathcal{R} \mathcal{T}$ (resp. $\mathcal{B} \mathcal{T}, \mathcal{T}_{0}$ ) whose objects are the same as those of $\mathcal{R} \mathcal{T}$, but whose Hom spaces will be quotients of the corresponding Hom spaces in $\mathcal{R} \mathcal{T}$ (resp. $\left.\mathcal{B} \mathcal{T}, \mathcal{T}_{0}\right)$. In general our categories will be multipolar. We refer to the case where there is just one pole on the left, as the affine case.

Definition 3.3. The multipolar Temperley-Lieb category $\mathcal{M T} \mathcal{L}(q)$ is the quotient category of $\mathcal{R} \mathcal{T}(q)$ obtained by imposing locally the skein relations (3.3) and (3.4) below.
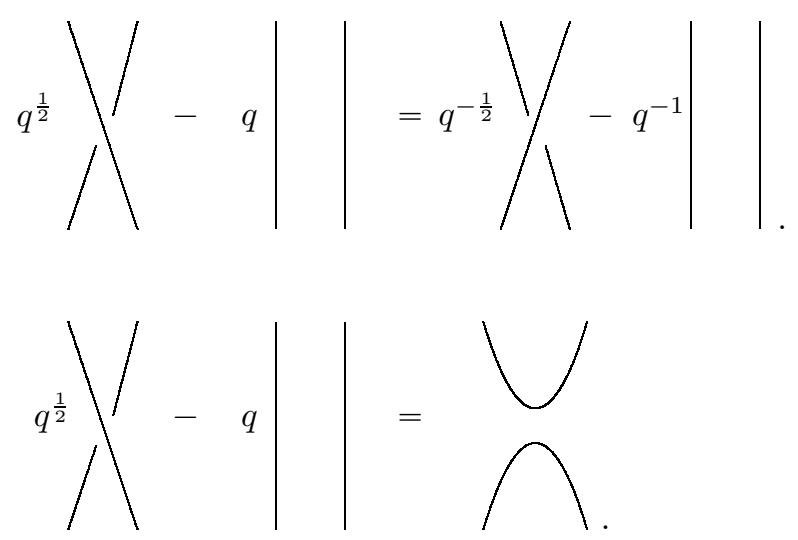

Lemma 3.4. In the category $\mathcal{M T} \mathcal{L}(q)$, we have the following identity.

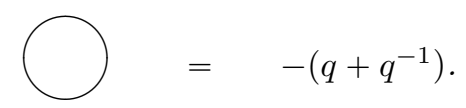

Proof. Let us write $C_{1}=q^{\frac{1}{2}} \sigma_{1}-q \in \operatorname{End}_{\mathcal{M} \mathcal{T} \mathcal{L}}\left(m, v^{2}\right)$. By (3.3), we have $q^{\frac{1}{2}} \sigma_{1}-q=$ $q^{-\frac{1}{2}} \sigma_{1}^{-1}-q^{-1}$, which is equivalent to

$$
\left(\sigma_{1}-q^{\frac{1}{2}}\right)\left(\sigma_{1}+q^{-\frac{3}{2}}\right)=0 .
$$

It follows that $C_{1}\left(\sigma_{1}+q^{-\frac{3}{2}}\right)=0$ and hence that $C_{1}^{2}=C_{1}\left(q^{\frac{1}{2}} \sigma_{1}-q\right)=C_{1}\left(-q^{-1}-q\right)$. But from (3.4) it is evident that $C_{1}^{2}=F \amalg C_{1}$, where $F$ is a free loop, and hence a morphism in $\operatorname{Hom}_{\mathcal{M} \mathcal{T}(q)}(0,0)=\mathcal{K}_{0}$. It follows that $F=-\left(q+q^{-1}\right)$ as claimed. 
Definition 3.5. If we impose the skein relations (3.3) and (3.4) on the subcategories $\mathcal{B} \mathcal{T}$ and $\mathcal{T}_{0}$, we obtain the affine Temperley-Lieb category $\mathcal{T} \mathcal{L}^{a}(q)$ and the Temperley-Lieb category $\mathcal{T} \mathcal{L}(q)$ respectively.

Remark 3.6. Note that $\mathcal{T} \mathcal{L}^{a}(q)$ and $\mathcal{T} \mathcal{L}(q)$ are also the full subcategories of $\mathcal{M T} \mathcal{L}(q)$ with objects $\left(m, v^{r}\right)$ and $v^{r}(r=0,1, \ldots)$ respectively. The identification of $\mathcal{T}_{0}$ with a subcategory of $\mathcal{B T}$ in Remark 3.2 leads to a similar identification of $\mathcal{T} \mathcal{L}(q)$ with the corresponding subcategory of $\mathcal{T} \mathcal{L}^{a}(q)$.

Proposition 3.7 identifies the endomorphism algebras in the categories above and provides a justification for the terminology above.

\section{Proposition 3.7.}

(1) For $r \geqslant 1$, the subalgebra of $\operatorname{End}_{\mathcal{T} \mathcal{L}^{a}(q)}\left(m, v^{r}\right)$ generated by the images of the elements $\xi_{1}$ and $\sigma_{i}(i=1,2, \ldots, r-1)$ depicted in Figure 5 is $\widehat{\mathrm{TL}}_{r}^{a}(q)$, the extended affine Temperley-Lieb algebra [14, Definition (4.1)].

(2) For $r \geqslant 0, \operatorname{End}_{\mathcal{T} \mathcal{L}(q)}\left(m, v^{r}\right) \cong \mathrm{TL}_{r}(q)$, the Temperley-Lieb algebra.

Proof. The algebra $\operatorname{End}_{\mathcal{R} \mathcal{T}}\left(m, v^{r}\right)$ contains a subalgebra generated by $\xi_{1}, \sigma_{1}, \ldots$, $\sigma_{r-1}$ subject to certain relations. We have seen in the proof of Lemma 3.4 that the relations (3.3) is equivalent to (3.6). Thus in case (1) the relevant algebra is a quotient of $\mathcal{K}_{0} \Gamma_{r} /\left\langle\left(q^{\frac{1}{2}} \sigma_{1}-q\right)\left(q^{\frac{1}{2}} \sigma_{1}+q^{-1}\right)\right\rangle$, where $\Gamma_{r}$ is the Artin braid group of type $B_{r}$. But by [14, Definition (3.1)], this is isomorphic to the extended affine Hecke algebra $\hat{H}_{r}^{a}(q)\left[22\right.$, since the generators $\sigma_{i}$ (of $\Gamma_{r}$ ) may be replaced by $q^{\frac{1}{2}} \sigma_{i}$ without changing the relations.

In case $(2)$ we obtain in a similar way that $\operatorname{End}_{\mathcal{T} \mathcal{L}(q)}\left(m, v^{r}\right)$ is a quotient of the algebra $\frac{\mathcal{K}_{0} \mathbb{B}_{r}}{\left\langle\left(q^{\frac{1}{2}} \sigma_{1}-q\right)\left(q^{\frac{1}{2}} \sigma_{1}+q^{-1}\right)\right\rangle}$, where $\mathbb{B}_{r}$ is the $r$-string braid group and this latter algebra is well known to be isomorphic to the Hecke algebra $H_{r}(q)$.

Now write $C_{i}=q^{\frac{1}{2}} \sigma_{i}-q$. It is clear from (3.4) by composing diagrams that $C_{i} C_{i+1} C_{i}=C_{i}$. Expressing this relation in terms of the Hecke algebra $H_{r}(q)$, which has basis elements $T_{w}, w \in \operatorname{Sym}_{r}$ (note that here $T_{w}$ is taken to be a word in the $\left.q^{\frac{1}{2}} \sigma_{i}\right)$, it follows that in $\operatorname{End}_{\mathcal{M T} \mathcal{L}(q)}\left(m, v^{r}\right)$, which is a quotient of $\widehat{H}_{r}^{a}(q)$, we have the relation $F_{i}=0$, where

$$
F_{i}=\sum_{w \in \mathrm{Sym}_{3}}\left(-q^{-1}\right)^{\ell(w)} T_{w}=0
$$

But $F_{i}$ is (a scalar multiple of) the transform of the element $E_{i}$ in 14, Definition (4.1)] under the automorphism of $\hat{H}_{r}^{a}(q)$ defined by $q^{\frac{1}{2}} \sigma_{i} \mapsto-q^{-\frac{1}{2}} \sigma_{i}^{-1}, \xi_{1} \mapsto \xi_{1}^{-1}$. The statement (1) now follows from loc. cit. and similarly for (2).

Remark 3.8. The tangle category $\mathcal{R} \mathcal{T}$ is remarkably rigid with respect to skein relations of the form (3.3) and (3.4). In fact the relations we have given may be shown to be essentially unique with respect to the requirement of compatibility with the sliding relations of Theorem $3.1(\mathrm{~d})$. 
Next consider in the category $\mathcal{M T} \mathcal{L}(q)$ the morphisms depicted in (3.7).
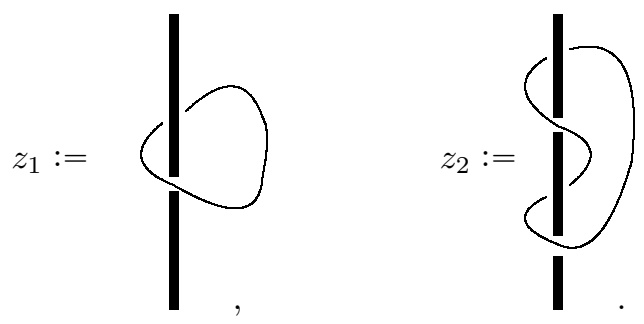

Lemma 3.9. The elements $z_{1}$ and $z_{2}$ are central in $\mathcal{M T} \mathcal{L}(q)$ in the following sense. Let $D$ be a diagram $D:\left(A_{1}, m, A_{2}\right) \rightarrow\left(B_{1}, m, B_{2}\right)$ in $\mathcal{M T} \mathcal{L}(q)$, where the two $m$ 's shown are connected by a thick arc. Then

$$
D\left(\operatorname{id}_{A_{1}} \otimes z_{i} \otimes \mathrm{id}_{A_{2}}\right)=\left(\operatorname{id}_{B_{1}} \otimes z_{i} \otimes \mathrm{id}_{B_{2}}\right) D, \quad i=1,2 .
$$

This is clear diagrammatically, because the thin loops may be thought of as pulled very tightly around the pole, so that they may be slid through any arcs entangling the pole. However, note from Remark 3.11(3) that $z_{1}$ and $z_{2}$ are not independent in $\mathcal{M T} \mathcal{L}(q)$.

The next result relates $z_{1}$ and $z_{2}$ to polar loops. It is important for subsequent developments.

Lemma 3.10. The following relation holds in $\mathcal{M T} \mathcal{L}(q)$, and hence also in $\mathcal{T} \mathcal{L}^{a}(q)$.

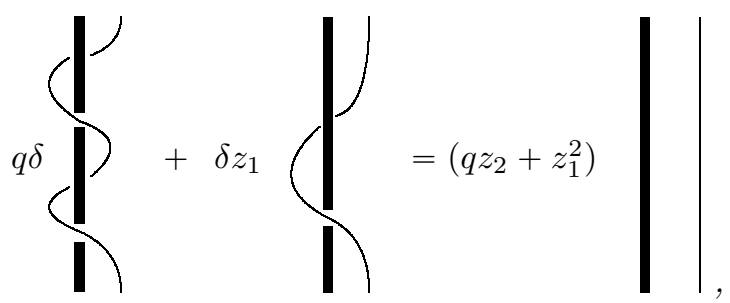

where the right hand side is a symbolic notation for $\left(q z_{2}+z_{1}^{2}\right) \otimes \mathrm{id}_{v}$.

Proof. We prove the relation diagrammatically. Consider the first relation of (3.1), that is, $\xi_{1} \sigma_{1} \xi_{1} \sigma_{1}=\sigma_{1} \xi_{1} \sigma_{1} \xi_{1}$, in $\Gamma_{2}$. Now the skein relations (3.3) and (3.4) imply that in the above notation, $q^{1 / 2} \sigma_{1}=q+C_{1}$. Hence substituting for $\sigma_{1}$, we obtain $\xi_{1}\left(C_{1}+q\right) \xi_{1}\left(C_{1}+q\right)=\left(C_{1}+q\right) \xi_{1}\left(C_{1}+q\right) \xi_{1}$, which after simplification yields the following relation in $\mathcal{M T} \mathcal{L}(q)$ :

$$
\xi_{1} C_{1} \xi_{1} C_{1}+q \xi_{1}^{2} C_{1}=C_{1} \xi_{1} C_{1} \xi_{1}+q C_{1} \xi_{1}^{2} .
$$

In terms of diagrams, this is expressed as follows.

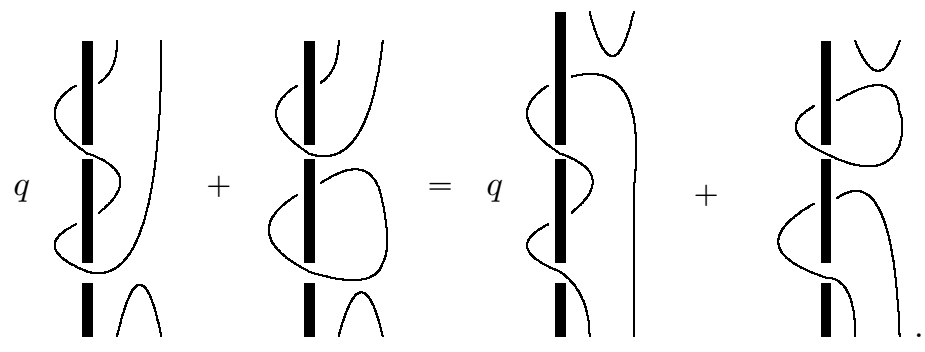


Now premultiply this equation by $\$, and then pull the bottom right end point of the string in each of the four diagrams anticlockwise to the top by using the straightening relation. One then obtains the stated relation.

Remark 3.11.

(1) The equation in Lemma 3.10 may be written symbolically as follows.

$$
\delta \xi_{1}^{2}=-q^{-1} \delta \xi_{1} z_{1} \otimes \mathrm{id}_{v}+z_{2} \otimes \mathrm{id}_{v}+q^{-1} z_{1}^{2} \otimes \mathrm{id}_{v} .
$$

(2) In principle, one might define elements $z_{i}$ for all $i \in \mathbb{Z}$, which are central in $\mathcal{M T} \mathcal{L}(q)$ in the sense above, where the loop of the thin arc in $z_{i}$ wraps around the pole $i$ times. However, if we apply the operator $D \mapsto A \circ\left(\xi_{1}^{i} \otimes\right.$ $I \circ D) \circ U$, where $A$ and $U$ are as in Figure 11, to the relation in the proof of Lemma 3.10, it is easily checked that we obtain

$$
q \delta z_{i+2}+\delta z_{1} z_{i+1}=\left(q z_{2}+z_{1}^{2}\right) z_{i}, \quad i \in \mathbb{Z},
$$

which uniquely determines all $z_{i}$ and $\left(q z_{2}+z_{1}^{2}\right)^{i} z_{-i}$ for $i \geqslant 0$ as polynomials in $z_{1}$ and $z_{2}$, with $z_{0}=\delta \mathrm{id}_{m}$.

(3) From the diagrams it is evident that $z_{-1}=z_{1}$. Hence putting $i=-1$ in (3.8) yields

$$
q \delta z_{1}+\delta^{2} z_{1}=\left(q z_{2}+z_{1}^{2}\right) z_{-1}=q z_{1} z_{2}+z_{1}^{3},
$$

which simplifies to $z_{1}\left(z_{1}^{2}+q z_{2}+q^{-1} \delta\right)=0$.

In constructing quotients of $\mathcal{M T} \mathcal{L}(q)$ by setting $z_{1}$ and $z_{2}$ equal to constants, this relation imposes constraints upon those constants. The values in (3.9) satisfy these constraints.

(4) Polynomials in $z_{ \pm i} \otimes \operatorname{id}_{v^{r}}$ (for $i \geqslant 1$ ) of degree greater than 0 are contained in $\operatorname{End}_{\mathcal{T} \mathcal{L}^{a}(q)}\left(m, v^{r}\right)$, but are not elements of the extended affine TemperleyLieb algebra $\widehat{\mathrm{TL}}_{r}^{a}(q)$.

3.4. Definition and structure of the category $\mathcal{T} \mathcal{L B}(q, \Omega)$. In this section we define (Definition 3.14) and study a category $\mathcal{T} \mathcal{L B}(q, \Omega)$ which will ultimately turn out to be isomorphic to $\mathbb{T} \mathbb{B}(q, Q)$ for appropriate parameter choices.

We begin by considering the quotient category of $\mathcal{M T} \mathcal{L}(q)$ obtained by identifying the central morphisms $z_{1}, z_{2}: m \rightarrow m$ (see (3.7)) with scalars, that is, by imposing conditions on the removal of loops which are entangled with the pole. A certain specialisation of this category is the category $\mathcal{T} \mathcal{L B}(q, \Omega)$.

Remark 3.12. Note that if $z_{1}$ and $z_{2}$ are identified with scalars, then Lemma 3.10 implies that in the resulting specialised (quotient) category, $\xi_{1}$ satisfies a quadratic relation. The apparently mysterious choice of parameters below is such that this quadratic relation reads $\left(q \xi_{1}-\Omega\right)\left(q \xi_{1}-\Omega^{-1}\right)=0$ (cf. Lemma 3.15). Note also that our choice of parameters is consistent with the constraint implied by Remark $3.11(3)$.

Definition 3.13. Fix an invertible scalar $\Omega \in \mathcal{K}_{0}$, and set

$$
a_{1}=-\left(\Omega+\Omega^{-1}\right), \quad a_{2}=-q^{-1}\left(\left(\Omega+\Omega^{-1}\right)^{2}+\delta q^{-1}\right) .
$$

Let $\mathcal{M T} \mathcal{L}(q, \Omega)$ be the category whose objects are the same as those of $\mathcal{M T} \mathcal{L}(q)$, and whose modules of morphisms are obtained from those in $\mathcal{M T} \mathcal{L}(q)$ by imposing 
all relations which are consequences of the two equalities

$$
z_{1}=a_{1} \mathrm{id}_{m}, \quad z_{2}=a_{2} \mathrm{id}_{m} .
$$

Definition 3.14. Let $\mathcal{T} \mathcal{L B}(q, \Omega)$ be the full subcategory of $\mathcal{M T} \mathcal{L}(q, \Omega)$ with objects $\left(m, v^{r}\right)$ for all $r \in \mathbb{Z}_{\geqslant 0}$.

This will be referred to as the Temperley-Lieb category of type $B$. The justification for the terminology will be provided by Theorem 3.27 .

The following relation is an immediate consequence of Lemma 3.10 . We refer to it as the third skein relation.

Lemma 3.15. The following skein relation holds in $\mathcal{M T} \mathcal{L}(q, \Omega)$, and hence also in $\mathcal{T} \mathcal{L B}(q, \Omega)$.

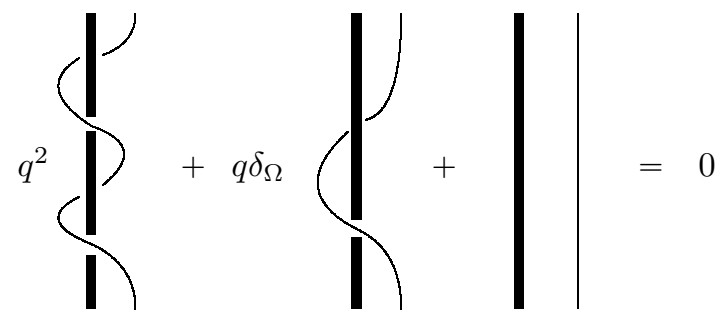

It is easy to see that the image of the Temperley-Lieb category $\mathcal{T} \mathcal{L}(q)$ in the quotient category $\mathcal{M T} \mathcal{L}(q, \Omega)$ is canonically isomorphic to itself. Thus we shall regard $\mathcal{T} \mathcal{L}(q)$ as a full subcategory of $\mathcal{M T} \mathcal{L}(q, \Omega)$.

The following result is clear.

Lemma 3.16. The tensor product functor on $\mathcal{R} \mathcal{T}$ induces a bi-functor

$$
\otimes: \mathcal{M T} \mathcal{L}(q, \Omega) \times \mathcal{M T} \mathcal{L}(q) \longrightarrow \mathcal{M T} \mathcal{L}(q, \Omega),
$$

which is defined on morphisms by juxtaposition of diagrams. It restricts to a bifunctor

$$
\otimes: \mathcal{T} \mathcal{L B}(q, \Omega) \times \mathcal{T} \mathcal{L}(q) \longrightarrow \mathcal{T} \mathcal{L} \mathcal{B}(q, \Omega)
$$

Remark 3.17. The Temperley-Lieb category $\mathcal{T} \mathcal{L}(q)$ may be thought of as a subcategory of $\mathcal{T} \mathcal{L B}(q, \Omega)$, since diagrams from $\left(v^{r}\right)$ to $\left(v^{s}\right)$ are evidently in bijection with diagrams $\left(m, v^{r}\right)$ to $\left(m, v^{s}\right)$ which have no entanglement with the pole. Thus $\mathcal{T} \mathcal{L}(q)$ is the subcategory of $\mathcal{T} \mathcal{L B}(q, \Omega)$ with precisely such morphisms.

We now investigate the structure of the Temperley-Lieb category $\mathcal{T} \mathcal{L B}(q, \Omega)$ in more depth. Morphisms of $\mathcal{T} \mathcal{L B}(q, \Omega)$ are linear combinations of diagrams with only one vertical thick arc placed at the left end, which can be described explicitly as follows.

Definition 3.18. Call a tangle diagram in $\mathcal{T} \mathcal{L B}(q, \Omega)$ a Temperley-Lieb diagram if it satisfies the following conditions:

(1) the diagram has only one vertical thick arc placed at the left end, which will be called the pole;

(2) there are no loops in the diagram;

(3) arcs do not self-tangle, and thin arcs do not tangle with thin arcs;

(4) if a thin arc tangles with the thick arc, it crosses the thick arc just twice, and crosses behind the pole in the upper crossing. 
For example, the diagrams in Figure 6] and Figure 7 are morphisms in $\mathcal{T} \mathcal{L B}(q, \Omega)$. The one in Figure 7 is not in the subcategory $\mathcal{T} \mathcal{L}(q)$ of $\mathcal{T} \mathcal{L B}(q, \Omega)$, while that in Figure 6 is in $\mathcal{T} \mathcal{L}(q)$.

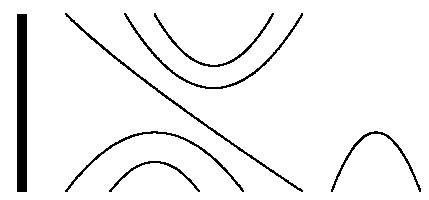

Figure 6. A diagram $\left(m, v^{7}\right) \rightarrow\left(m, v^{5}\right)$

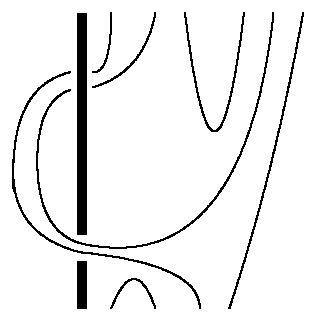

Figure 7. A diagram $\left(m, v^{4}\right) \rightarrow\left(m, v^{6}\right)$

Figure 6 is a morphism $\left(m, v^{7}\right) \rightarrow\left(m, v^{5}\right)$, where arcs do not cross, and Figure 7 is a morphism $\left(m, v^{4}\right) \rightarrow\left(m, v^{6}\right)$, where 2 thin arcs over cross the thick arc twice each. Note that there is a unique Temperley-Lieb diagram $m \rightarrow m$ consisting of a thick arc only.

The spaces of morphisms of $\mathcal{T} \mathcal{L B}(q, \Omega)$ are easily seen to be spanned by Temperley-Lieb diagrams, since the relations in Section 3.3 may be used to reduce any diagram to a linear combination of Temperley-Lieb diagrams.

Composition of morphisms may be described as follows. Given morphisms $D$ : $B \rightarrow T$ and $D^{\prime}: T \rightarrow U$ represented by Temperley-Lieb diagrams, their composition is defined by the following steps

(1) Concatenation of diagrams. Concatenate the diagrams $D$ and $D^{\prime}$ by joining the points on the top of $D$ with those on the bottom of $D^{\prime}$.

(2) Reduction to Temperley-Lieb diagrams. Apply locally the skein relation (Lemma 3.15), free loop removal (3.5) and tangled loop removal (Definition (3.13) to turn the resulting diagram into a linear combination of TemperleyLieb diagrams $B \rightarrow U$.

(3) The result of step (2) is the composition $D^{\prime} \circ D$ of the morphisms $D$ and $D^{\prime}$.

The following result is obtained by repeatedly applying the straightening relations.

Lemma 3.19. Let $N$ be any non-negative integer. Then for $r=0,1, \ldots, 2 N$, the vector spaces $\operatorname{Hom}_{\mathcal{T} \mathcal{L B}(q, \Omega)}\left(\left(m, v^{r}\right),\left(m, v^{2 N-r}\right)\right)$ are all isomorphic.

Proof. The proof is the same as in [20]. It amounts to simply observing that by pulling the rightmost top arc of a diagram in $\operatorname{Hom}_{\mathcal{T} \mathcal{L B}(q, \Omega)}\left(\left(m, v^{r}\right),\left(m, v^{2 N-r}\right)\right)$ 
down, one obtains a diagram in $\operatorname{Hom}_{\mathcal{T} \mathcal{L B}(q, \Omega)}\left(\left(m, v^{r+1}\right),\left(m, v^{2 N-r-1}\right)\right)$, and this procedure defines the required isomorphism.

A special case of the above is $W(2):=\operatorname{Hom}_{\mathcal{T} \mathcal{L B}(q, \Omega)}\left(m,\left(m, v^{2 N}\right)\right)$. Let $F_{t} W(2 N)$ be the subspace of $W(2 N)$ spanned by diagrams such that at most $t$ thin arcs are entangled with the pole. For example, Figure 8 is a diagram $m \rightarrow\left(m, v^{12}\right)$ which

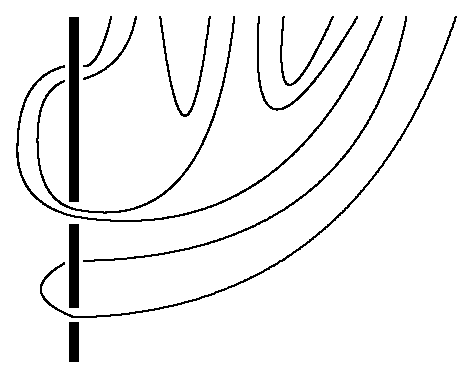

FiguRE 8. A diagram $m \rightarrow\left(m, v^{12}\right)$

lies in $F_{3} W(12)$. Note that two of the three arcs which cross the pole at its upper part are "parallel" when they cross the pole. Let us make this notion more precise.

Definition 3.20. Any thin arc which is entangled with the pole has two polar crossings, and in this way defines an interval on the pole, which we call its "polar interval". We say that two thin arcs in a Temperley-Lieb diagram are parallel if they are both entangled with the pole and the polar interval defined by one is contained in the polar interval defined by the other.

Remark 3.21. There is a partial order on the thin arcs in a Temperley-Lieb diagram which are entangled with the pole, which is defined by containment of their corresponding polar intervals. Two such arcs are parallel if they are comparable in this partial order.

Definition 3.22. Call a Temperley-Lieb diagram $m \rightarrow\left(m, v^{2 N}\right)$ distinguished if any pair of thin arcs which entangle the pole are parallel, and standard if no two thin arcs tangling with the pole are parallel. In view of Remark 3.21, a diagram is distinguished (resp. standard) if those among its arcs which entangle the pole are totally ordered (resp. have no two arcs which are comparable) in the partial order on pole-entangled arcs.

Examples of distinguished and standard diagrams are given respectively in Figure 9 and in Figure 10 .

It is easily seen that there is a unique distinguished (resp. standard) TemperleyLieb diagram $m \rightarrow\left(m, v^{2 N}\right)$ with $N$ thin arcs tangling with the pole.

Recall that $F_{t} W(2 N)$ is the subspace of $W(2 N)=\operatorname{Hom}_{\mathcal{T} \mathcal{L B}(q, \Omega)}\left(m,\left(m, v^{2 N}\right)\right)$ spanned by diagrams with at most $t$ thin arcs which are entangled with the pole.

\section{Lemma 3.23.}

(1) Any Temperley-Lieb diagram in $F_{t} W(2 N)$ not belonging to $F_{t-1} W(2 N)$ can be expressed as a linear combination of elements of $F_{t-1} W(2 N)$ and a distinguished (resp. standard) diagram with t thin arcs tangling with the pole, where the coefficient of the distinguished (resp. standard) diagram is a (half-integer) power of $q$. 


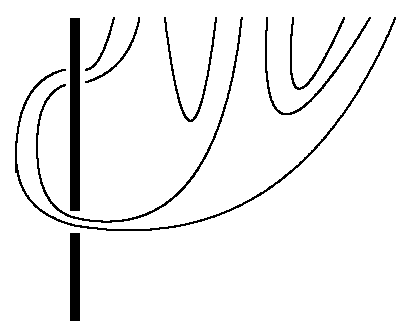

Figure 9. A distinguished diagram $m \rightarrow\left(m, v^{10}\right)$

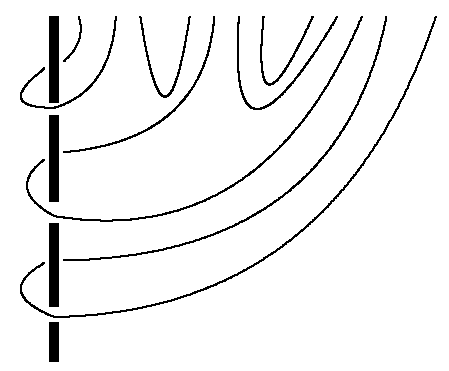

Figure 10. A standard diagram $m \rightarrow\left(m, v^{12}\right)$

(2) Any Temperley-Lieb diagram in $F_{t} W(2 N)$ can be expressed as a linear combination of distinguished (resp. standard) diagrams.

Proof. By stretching the entangled arcs, one sees that any Temperley-Lieb diagram $m \rightarrow\left(m, v^{2 N}\right)$ with $t$ thin arcs entangling the pole can be expressed as the composition $D^{\prime} \circ D$ of two diagrams $D: m \rightarrow\left(m, v^{2 t}\right)$ and $D^{\prime}:\left(m, v^{2 t}\right) \rightarrow\left(m, v^{2 N}\right)$, where $D^{\prime}$ has no thin arcs which entangle the pole.

Now consider a diagram $D: m \rightarrow\left(m, v^{2 t}\right)$ with $t$ thin arcs entangling the pole. If $D$ is distinguished, then the original diagram is distinguished. If $D$ is not distinguished, we may pull the thin arcs one by one so that all $t$ arcs are parallel, at the expense of introducing crossings, which after sliding (cf. Theorem 3.1(3(d))), will all be to the right of the pole. We now use skein relations to remove the crossings among the thin arcs. This leads to a linear combination of diagrams, where the only diagram with $t$ thin arcs entangling the pole is the distinguished one, and its coefficient is a power of $q$.

In a similar way, we can express any Temperley-Lieb diagram in $F_{t} W(2 N)$ as a linear combination of a standard diagram and diagrams in $F_{W} t-1(2 N)$. This proves part (1) of the Lemma.

To see (2), observe that given any Temperley-Lieb diagram $m \rightarrow\left(m, v^{2 N}\right)$ with $t$ thin arcs entangling the pole, we may use part (1) to express it as a linear combination of a distinguished (resp. standard) diagram and diagrams in $F_{t-1} W(2 N)$. We then repeat the reduction process for diagrams in $F_{t-1} W(2 N)$, then $F_{t-2} W(2 N)$, etc., and after $t$ iterations, arrive at the statement (2).

We have the following result. 
Lemma 3.24. For all $r=0,1, \ldots, 2 N$,

$$
\operatorname{dim} \operatorname{Hom}_{\mathcal{T} \mathcal{L B}(q, \Omega)}\left(\left(m, v^{r}\right),\left(m, v^{2 N-r}\right)\right)=\left(\begin{array}{c}
2 N \\
N
\end{array}\right) .
$$

Proof. We give a proof here which will be useful for proving Theorem 4.9 By Lemma 3.19, we only need to prove the dimension formula for $r=0$. Recall that in the proof of Lemma 3.23 we introduced the following filtration for $W(2 N)=$ $\operatorname{Hom}_{\mathcal{T} \mathcal{L B}(q, \Omega)}\left(m,\left(m, v^{2 N}\right)\right)$.

(3.10) $F_{N} W(2 N) \supset F_{N-1} W(2 N) \supset \cdots \supset F_{1} W(2 N) \supset F_{0} W(2 N) \supset \varnothing$,

where $F_{t} W(2 N)$ is the subspace spanned by Temperley-Lieb diagrams with at most $t$ thin arcs entangled with the pole. Let $\operatorname{End}(2 N)=\operatorname{Hom}_{\mathcal{T} \mathcal{L B}(q, \Omega)}\left(\left(m, v^{2 N}\right)\right.$, $\left.\left(m, v^{2 N}\right)\right)$ and denote by $\operatorname{End}^{0}(2 N)$ the subspace of $\operatorname{End}(2 N)$ spanned by diagrams without tanglement. Then $\operatorname{End}^{0}(2 N)$ is a subalgebra of $\operatorname{End}(2 N)$ isomorphic to the Temperley-Lieb algebra $\mathrm{TL}_{2 N}(q)$ of degree $2 N$. Now $\operatorname{End}^{0}(2 N)$ acts naturally on $W(2 N)$, and the $F_{t} W(2 N)$ are $\operatorname{End}^{0}(2 N)$-submodules. Clearly $\frac{F_{t} W(2 N)}{F_{t-1} W(2 N)}$ is isomorphic to a cell module $W_{2 t}(2 N)$ [13, Def. $\left.(2.2)\right]$ for $\left.\operatorname{End}^{0}(2 N)\right)$, which is a simple module in the present generic context. Hence as vector space,

$$
\operatorname{Hom}_{\mathcal{T} \mathcal{L B}(q, \Omega)}\left(m,\left(m, v^{2 N}\right)\right) \cong \bigoplus_{t=0}^{N} W_{2 t}(2 N) .
$$

Using the well-known fact [15] that

$$
\operatorname{dim} W_{2 t}(2 N)=\left(\begin{array}{c}
2 N \\
N-t
\end{array}\right)-\left(\begin{array}{c}
2 N \\
N-t-1
\end{array}\right)
$$

with $\operatorname{dim} W_{2 N}(2 N)=\left(\begin{array}{c}2 N \\ 0\end{array}\right)=1$, we obtain the result.

3.5. Standard diagrams, tensor product and generators. Recall from Remark 3.17 that the diagrams in the subcategory $\mathcal{T} \mathcal{L}(q)$ of $\mathcal{T} \mathcal{L B}(q, \Omega)$ are those which involve no entanglement with the pole. As such, they look like a disentangled pole adjacent to a 'usual' finite Temperley-Lieb diagram (cf. [13, 14]). Now it follows from Lemma 3.16 that there is an obvious functor

$$
\mathcal{T} \mathcal{L} \mathcal{B}(q, \Omega) \times \mathcal{T} \mathcal{L}(q) \longrightarrow \mathcal{T} \mathcal{L} \mathcal{B}(q, \Omega)
$$

which is defined by juxtaposition of diagrams, where the disentangled pole is omitted from the second factor. Moreover it is clear that the diagrams depicted as $I, A, U$ in Figure 11 generate the subcategory $\mathcal{T} \mathcal{L}(q)$ under composition and the tensor product defined by (3.12).
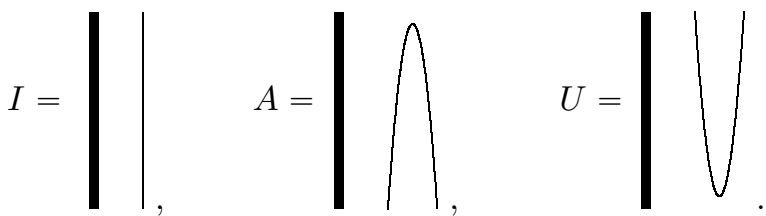

FIGURE 11. Generators of the subcategory $\mathcal{T} \mathcal{L}(q)$ 
All relations among these generators are well known to be consequences of the following.

$$
A U=\delta_{q}=-\left(q+q^{-1}\right) ; \quad(A \otimes I)(I \otimes U)=I .
$$

We wish to determine a similar presentation for the whole of $\mathcal{T} \mathcal{L B}(q, \Omega)$. For this we begin by observing that any Temperley-Lieb diagram may be expressed as a linear combination of diagrams which are standard in the following sense.

Definition 3.25. A Temperley-Lieb diagram $D:\left(m, v^{r}\right) \rightarrow\left(m, v^{s}\right)$ is called standard if $\left(D \otimes \mathrm{id}_{v^{r}}\right)\left(\mathrm{id}_{m} \otimes \mathbb{U}_{r}\right): m \rightarrow\left(m, v^{r+s}\right)$ is a standard diagram in the sense of Definition 3.22, where $\mathbb{U}_{r}: \varnothing \rightarrow v^{2 r}$ is given by the following diagram.

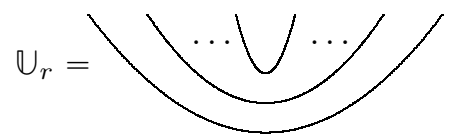

It follows from part (2) of Lemma 3.23 that

Corollary 3.26. Any Temperley-Lieb diagram can be expressed as a linear combination of standard diagrams.

It is now clear that to obtain $\mathcal{T} \mathcal{L B}(q, \Omega)$ from $\mathcal{T} \mathcal{L}(q)$ just one extra generator, depicted $L$ in the diagram below, needs to be added to the set of generators of $\mathcal{T} \mathcal{L}(q)$ given in Figure 11, Note that $L$ is the image of $\xi_{1}$ in $\mathcal{T} \mathcal{L B}(q)$.

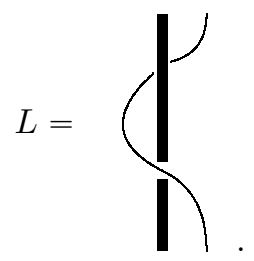

We shall use these generators in the proof of Theorem 3.27 .

Note that among the relations in $\mathcal{T} \mathcal{L B}(q, \Omega)$ we have, using Lemma 3.10 and Definition 3.13 .

$$
\begin{array}{r}
q L^{2}=\left(\Omega+\Omega^{-1}\right) L-q^{-1} I, \\
A(L \otimes I) U=-\left(\Omega+\Omega^{-1}\right) .
\end{array}
$$

3.6. An equivalence of categories. We have defined two "Temperley-Lieb categories of type $B$ ", viz. the category $\mathcal{T} \mathcal{L B}(q, \Omega)$ of Definition 3.13 and the category $\mathbb{T} \mathbb{B}(q, Q)$ of Definition 2.1. Both categories contain the finite Temperley-Lieb category $\mathcal{T} \mathcal{L}(q)$ as a subcategory. In the case of $\mathbb{T} \mathbb{B}(q, Q)$ this is realised as in Section 2.2. In the case of $\mathcal{T} \mathcal{L B}(q, \Omega)$ (cf. Definition 3.13), $\mathcal{T} \mathcal{L}(q)$ may be thought of as having the same objects $\left\{\left(m, v^{r}\right)\right\}, r=0,1, \ldots$ as $\mathcal{T} \mathcal{L B}(q, \Omega)$, but where the morphisms are linear combinations of tangles which are not entwined with the pole.

Our next objective is to prove the following result.

Theorem 3.27. Let $R$ be an integral domain with invertible elements $q, Q$ and $\Omega$ and an element $\sqrt{-1}$ such that $\sqrt{-1}^{2}=-1$. Then there is an isomorphism of categories $\mathcal{M}: \mathbb{T} \mathbb{B}(q, Q) \longrightarrow \mathcal{T} \mathcal{L} \mathcal{B}(q, \Omega)$ which takes the object $r \in \mathbb{Z}$ to $\left(m, v^{r}\right)$, is the 
identity on $\mathcal{T} \mathcal{L}(q)$, and respects the tensor product, if and only if $\Omega= \pm(\sqrt{-1} Q)^{ \pm 1}$. In this case we have $\mathcal{M}\left(C_{0}\right)=\sqrt{-1} q L-Q I$.

Note that the stated conditions on $\mathcal{M}$ imply that for diagrams $D \in \mathbb{T} \mathbb{B}(q, Q)$ and $D^{\prime} \in \mathcal{T} \mathcal{L}(q)$, we have

$$
\mathcal{M}\left(D \otimes D^{\prime}\right)=\mathcal{M}(D) \otimes D^{\prime}
$$

Proof of Theorem 3.27. We shall define $\mathcal{M}$ on the generators $A, U, I$ and $C_{0}$, the effect of $\mathcal{M}$ on objects having been given. Since $\mathcal{M}$ is to be the identity functor on the subcategory $\mathcal{T} \mathcal{L}(q)$, evidently we must have $\mathcal{M}(A)=A, \mathcal{M}(U)=U$ and $\mathcal{M}(I)=I$, where on the left side of these equations $A, U$ and $I$ are as in Figure 4, while on the right side they are as defined in Figure 11.

It remains only to define $\mathcal{M}\left(C_{0}\right)$. This is a morphism in $\operatorname{End}_{\mathcal{T} \mathcal{L B}(q, \Omega)}(m, v)$, and since this space has basis $I, L$, it follows that

$$
\mathcal{M}\left(C_{0}\right)=a L+b I,
$$

for $a, b \in R$. We shall determine constraints on $a, b$. First, observe that it follows by applying $A(-\otimes I) U$ to both sides of (3.17) that

$$
\mathcal{M}\left(A\left(C_{0} \otimes I\right) U\right)=a A(L \otimes I) U+b A(I \otimes I) U,
$$

whence using (3.15) and (2.1) (ii) it follows that

$$
\frac{q}{Q}+\frac{Q}{q}=a \delta_{\Omega}+b \delta_{q},
$$

where, for any invertible $x \in R, \delta_{x}=-\left(x+x^{-1}\right)$.

Next, we square both sides of (3.17) using the relations (3.14) and (2.1)(iii). One obtains

$$
\left(2 a b-q^{-1} \delta_{\Omega} a^{2}\right) L+\left(b^{2}-q^{-2} a^{2}\right)=a \delta_{Q} L+b \delta_{Q} I,
$$

and equating the coefficients of $L$ and $I$ respectively, we obtain

$$
\begin{array}{r}
2 a b-q^{-1} \delta_{\Omega} a^{2}=a \delta_{Q}, \\
b^{2}-q^{-2} a^{2}=b \delta_{Q} .
\end{array}
$$

Moreover, since evidently $a \neq 0$, we may divide (3.20) by $a$ to obtain

$$
2 b-q^{-1} \delta_{\Omega} a=\delta_{Q} .
$$

It therefore remains to solve equations (3.18), (3.21) and (3.22) for $a$ and $b$.

It is straightforward to show that (3.22) and (3.18) imply that

$$
b=-Q \text {. }
$$

It now follows easily from (3.18) that

$$
a=\frac{q\left(Q^{-1}-Q\right)}{\delta_{\Omega}} .
$$

Now the values of $a, b$ in (3.23) and (3.24) are easily shown to satisfy (3.18) and (3.22). However they satisfy (3.21) if and only if $\delta_{\Omega}^{2}=-\left(Q^{-1}-Q\right)^{2}$, and this holds if and only if

$$
\Omega= \pm \sqrt{-1} Q^{ \pm 1} .
$$

Substituting this into (3.24), we obtain

$$
a= \pm \sqrt{-1} q .
$$


It is now easily checked that the defining relations among the generators $A, U, I$ and $C_{0}$, which are those involving only $A, U$ and $I$, as well as those in (2.1), are respected by $\mathcal{M}$. Thus $\mathcal{M}$ is well defined; that is, $\mathcal{M}(\phi)$ is well defined for any morphism $\phi \in \mathbb{T} \mathbb{B}(q, Q)$.

But evidently, given the relation $\delta_{\Omega}^{2}=-\left(Q^{-1}-Q\right)^{2}, \mathcal{M}$ has an inverse functor, $\mathcal{M}^{-1}$ which is defined on the generators of $\mathcal{T} \mathcal{L}(q, \Omega)$ by $\mathcal{M}^{-1}: I \mapsto I ; \quad A \mapsto$ $A ; \quad U \mapsto U$ and $\mathcal{M}^{-1}(L)=a^{-1} C_{0}-a^{-1} b I$, where $a^{-1}= \pm \sqrt{-1} q^{-1}$ and $b=$ $\pm \sqrt{-1} \Omega^{ \pm 1}$. If we take $\Omega=\sqrt{-1} Q$ and $a=\sqrt{-1} q$ then $\mathcal{M}^{-1}(L)=-\sqrt{-1} q^{-1} C_{0}-$ $q^{-1} \Omega I$. It is now clear that provided $\delta_{\Omega}^{2}=-\left(Q^{-1}-Q\right)^{2}, \mathcal{M} \circ \mathcal{M}^{-1}=\operatorname{id}_{\mathcal{T} \mathcal{L B}(q, \Omega)}$ and $\mathcal{M}^{-1} \circ \mathcal{M}=\operatorname{id}_{\mathbb{T}\llcorner\mathbb{B}(q, Q)}$, proving the required isomorphism of categories.

Remark 3.28. It is evident from Theorem 3.27 that the Hom spaces in $\mathcal{T} \mathcal{L}(q, \Omega)$ and $\mathbb{T} \mathbb{B}(q, Q)$ have the same dimension. Hence Lemma 3.24 follows from Proposition 2.2. We have included an independent proof of this result to illustrate the role of the filtrations by tangle diagrams in the context of the latter category.

\section{Quantum Schur-Weyl DUality}

4.1. The quantum group $\mathrm{U}_{q}\left(\mathfrak{s l}_{2}\right)$. We briefly describe the representations and the universal $R$-matrix of $\mathrm{U}_{q}\left(\mathfrak{s l}_{2}\right)$ in this section. We write $\mathrm{U}_{q}$ for the $\mathcal{K}_{0}$-algebra $\mathrm{U}_{q}\left(\mathfrak{s l}_{2}\right)$. This has generators $E, F$ and $K^{ \pm 1}$, with relations

$$
K E K^{-1}=q^{2} E, \quad K F K^{-1}=q^{-2} F, \quad E F-F E=\frac{K-K^{-1}}{q-q^{-1}} .
$$

The comultiplication is given by

$$
\Delta(K)=K \otimes K, \quad \Delta(E)=E \otimes K+1 \otimes E, \quad \Delta(F)=F \otimes 1+K^{-1} \otimes F,
$$

and the antipode by

$$
S(E)=-E K^{-1}, \quad S(F)=-K F, \quad S(K)=K^{-1} .
$$

4.1.1. Representations. Projective modules. An integral weight $\mathrm{U}_{q}\left(\mathfrak{s l}_{2}\right)$-module $M$ of type-1 is one such that $M=\oplus_{k \in \mathbb{Z}} M_{k}$ where $M_{k}=\left\{v \in M K v=q^{k} v\right\}$. Let $\mathrm{U}_{q}(\mathfrak{b})$ be the Borel subalgebra of $\mathrm{U}_{q}\left(\mathfrak{s l}_{2}\right)$ generated by $E$ and $K^{ \pm 1}$. The category $\mathcal{O}_{\text {int }}$ is defined as the category of $\mathrm{U}_{q}\left(\mathfrak{s l}_{2}\right)$-modules $M$, which satisfy:

- $M$ is finitely generated as a $\mathrm{U}_{q}\left(\mathfrak{s l}_{2}\right)$-module.

- $M$ is locally $\mathrm{U}_{q}(\mathfrak{b})$ finite.

- $M$ is an integral weight module of type $\mathbf{1}$.

For any integer $\ell \in \mathbb{Z}$, denote by $\left(\mathcal{K}_{0}\right)_{\ell}=\mathcal{K}_{0} v^{+}$the 1-dimensional $\mathrm{U}_{q}(\mathfrak{b})$-module such that $E v^{+}=0$ and $K v^{+}=q^{\ell} v^{+}$, and let $M(\ell)=\mathrm{U}_{q}\left(\mathfrak{s l}_{2}\right) \otimes_{\mathrm{U}_{q}(\mathfrak{b})}\left(\mathcal{K}_{0}\right)_{\ell}$. This is the Verma module with highest weight $\ell$, which has a unique simple quotient $V(\ell)$. Then $M(\ell)$ and $V(\ell)$ are the standard and simple objects in $\mathcal{O}_{\text {int }}$. The simple module $V(\ell)$ is finite dimensional if and only if $\ell \geqslant 0$, and in this case it is $(\ell+1)$-dimensional.

The element $z:=F E+\frac{q K+q^{-1} K^{-1}}{\left(q-q^{-1}\right)^{2}}$ is central in the quantum group $\mathrm{U}_{q}\left(\mathfrak{s l}_{2}\right)$. It acts as the scalar $\chi_{\ell}:=\frac{q^{\ell+1}+q^{-\ell-1}}{\left(q-q^{-1}\right)^{2}}$ on any highest weight vector of weight $\ell$ and since it is central, therefore acts as the scalar $\chi_{\ell}$ on the whole of any highest weight module in $\mathcal{O}_{\text {int }}$ with highest weight $\ell$.

For any module $M \in \mathcal{O}_{\text {int }}$, if we define $M^{\chi \ell}:=\left\{m \in M \mid\left(z-\chi_{\ell}\right)^{i} m=\right.$ 0 for some $i \geqslant 0\}$, then $M^{\chi \ell}$ is a direct summand of $M$. We denote by $\mathcal{O}_{\text {int }}^{\chi \ell}$ the full 
subcategory of $\mathcal{O}_{\text {int }}$ whose objects are modules $M$ such that $M=M^{\chi \ell}$, and call it the block (which is indeed a block) of $\mathcal{O}_{\text {int }}$ corresponding to $\chi_{\ell}$. It follows from the definition of $\mathcal{O}_{\text {int }}$ that any $M \in \mathcal{O}_{\text {int }}$ is a direct sum of finitely many summands, each of which belongs to a block.

Evidently $\chi_{\ell}=\chi_{\ell^{\prime}}$ if and only if $\ell=\ell^{\prime}$ or $\ell+\ell^{\prime}+2=0$.

Definition 4.1. The weights $\ell, \ell^{\prime} \in \mathbb{Z}$ are said to be linked if $\ell=\ell^{\prime}$ or $\ell+\ell^{\prime}+2=0$. The linkage principal asserts here merely that $\chi_{\ell}=\chi_{\ell^{\prime}} \Longleftrightarrow \ell, \ell^{\prime}$ are linked.

Observe that if $\ell \geqslant-1$, then there exists no $\ell^{\prime}>\ell$ linked to $\ell$. This leads to the following result, which is well known, but we provide a proof for the convenience of the reader.

Lemma 4.2. Fix an integer $\ell \geqslant-1$.

(1) The Verma module $M(\ell)$ is projective in $\mathcal{O}_{\text {int }}$.

(2) If $M$ is a finite dimensional module in $\mathcal{O}_{\text {int }}$, then there is a linear isomorphism

$$
\operatorname{Hom}_{\mathrm{U}_{q}\left(\mathfrak{s l}_{2}\right)}(M(\ell), M(\ell) \otimes M) \cong M_{0},
$$

where $M_{0}$ is the zero weight space of $M$.

Proof. Consider first part (1). Let $\psi: M \rightarrow N$ be any surjection in $\mathcal{O}_{\text {int }}$. Then $\psi(M)=N$, so that $\psi\left(M^{\chi \ell}\right)=N^{\chi \ell}$. If $\phi: M(\ell) \longrightarrow N$ is any homomorphism, then clearly $\phi(M(\ell)) \subseteq N^{\chi_{\ell}}$ and hence to prove (1), we may suppose that $M$ and $N$ are in the block $\mathcal{O}_{\text {int }}^{\text {Xe }}$ of $\mathcal{O}_{\text {int }}$. Since the image $\phi\left(m_{+}\right)$of the highest weight vector $m_{+}$of $M(\ell)$ is in $N=\psi(M), \phi\left(m_{+}\right)=\psi(v)$ for some $v \in M$. Writing $v=v_{0}+v_{1}+\cdots+v_{k}$, where $v_{0}$ has weight $\ell$ and for $i \geqslant 1, v_{i}$ has weight $\ell_{i} \neq \ell$ (the $\ell_{i}$ being pairwise distinct), we see that for $j=1,2,3, \ldots$, we have $q^{j \ell}\left(\psi(v)-\psi\left(v_{0}\right)\right)+q^{j \ell_{1}} \psi\left(v_{1}\right)+$ $\cdots+q^{j \ell_{k}} \psi\left(v_{k}\right)=0$. A van der Monde type argument shows that $\psi\left(v_{i}\right)=0$ for $i>0$ and that $\psi(v)=\psi\left(v_{0}\right)$. Replacing $v$ by $v_{0}$, we may therefore assume that $v$ is a weight vector of weight $\ell$.

Now the subspace $\mathrm{U}_{q}(\mathfrak{b}) v \subseteq M$ contains a highest weight vector $v^{\prime}$ of weight (say) $\ell^{\prime}$. Thus $v^{\prime}$ is an eigenvector of $z$, with eigenvalue $\chi_{\ell^{\prime}}$. Since $M$ is in the block $\mathcal{O}_{i n t}^{\chi \ell}$, we must have $\chi_{\ell}=\chi_{\ell^{\prime}}$, i.e. $\ell^{\prime}$ is linked to $\ell$. But $\ell^{\prime} \geqslant \ell \geqslant-1$, so $\ell^{\prime}=\ell$, and hence $v$ is a highest weight vector in $M$.

It follows that the unique homomorphism $\phi^{\prime}: M(\ell) \longrightarrow M$ with $\phi^{\prime}\left(m_{+}\right)=v$, renders the following diagram commutative.

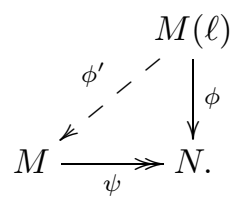

This proves that $M(\ell)$ is projective in $\mathcal{O}_{\text {int }}$.

We now prove part (2). Since the module $M$ given in part (2) is finite dimensional, $M(\ell) \otimes M=\mathrm{U}_{q}\left(\mathfrak{s l}_{2}\right) \otimes_{\mathrm{U}_{q}(\mathfrak{b})}\left(\left(\mathcal{K}_{0}\right)_{\ell} \otimes M\right)$. Applying the induction functor to the composition series of $\left(\mathcal{K}_{0}\right)_{\ell} \otimes M$ as $\mathrm{U}_{q}(\mathfrak{b})$-module leads to a filtration

$$
M(\ell) \otimes M:=W_{0} \supset W_{1} \supset W_{2} \supset \cdots \supset W_{D-1} \supset W_{D}=0, \quad D=\operatorname{dim} M,
$$

where the $W_{i}$ are in $\mathcal{O}_{i n t}$ and $W_{i} / W_{i+1}=M\left(\ell_{i}\right)$ for some integer $\ell_{i}$. By the projectivity of $M(\ell)$, we have

$$
\operatorname{Hom}_{\mathrm{U}_{q}\left(\mathfrak{s l}_{2}\right)}\left(M(\ell), W_{i}\right)=\operatorname{Hom}_{\mathrm{U}_{q}\left(\mathfrak{s l}_{2}\right)}\left(M(\ell), M\left(\ell_{i}\right)\right) \oplus \operatorname{Hom}_{\mathrm{U}_{q}\left(\mathfrak{s l}_{2}\right)}\left(M(\ell), W_{i+1}\right)
$$


as vector space, and hence

$$
\operatorname{Hom}_{\mathrm{U}_{q}\left(\mathfrak{s l}_{2}\right)}(M(\ell), M(\ell) \otimes M)=\bigoplus_{i} \operatorname{Hom}_{\mathrm{U}_{q}\left(\mathfrak{s l}_{2}\right)}\left(M(\ell), M\left(\ell_{i}\right)\right) .
$$

But $\operatorname{Hom}_{\mathrm{U}_{q}\left(\mathfrak{s}_{2}\right)}\left(M(\ell), M\left(\ell_{i}\right)\right) \neq 0$ only when $\ell$ and $\ell_{i}$ are linked. The condition $\ell \geqslant-1$ requires $\ell_{i}=\ell$, and in this case, $\operatorname{Hom}_{\mathrm{U}_{q}\left(\mathfrak{s}_{2}\right)}\left(M(\ell), M\left(\ell_{i}\right)\right)$ is one dimensional. Since the weight space of weight $\ell$ in $M(\ell) \otimes M$ is $v_{+} \otimes M_{0}$, we have $\operatorname{Hom}_{\mathrm{U}_{q}\left(\mathfrak{s l}_{2}\right)}(M(\ell), M(\ell) \otimes M) \cong M_{0}$.

Some properties of $V=V(1)$. There exists a basis $\left\{v_{1}, v_{-1}\right\}$ of $V$ such that the corresponding representation is given by

$$
K \mapsto\left(\begin{array}{cc}
q & 0 \\
0 & q^{-1}
\end{array}\right), \quad E \mapsto\left(\begin{array}{cc}
0 & 1 \\
0 & 0
\end{array}\right), \quad F \mapsto\left(\begin{array}{ll}
0 & 0 \\
1 & 0
\end{array}\right) .
$$

We have $V \otimes V=V(2) \oplus V(0)$, with the 1-dimensional submodule spanned by

$$
c_{0}:=-q v_{1} \otimes v_{-1}+v_{-1} \otimes v_{1} .
$$

Since $V$ is self-dual, there exists a unique (up to scalar multiple) non-degenerate invariant bilinear form $():, V \times V \longrightarrow \mathcal{K}_{0}$ given by

$$
\left(v_{1}, v_{-1}\right)=-q\left(v_{-1}, v_{1}\right)=1, \quad\left(v_{1}, v_{1}\right)=\left(v_{-1}, v_{-1}\right)=0 .
$$

Here invariance of the form means that $(X v, w)=(v, S(X) w)$ for all $v, w \in V$ and $X \in \mathrm{U}_{q}\left(\mathfrak{s l}_{2}\right)$, where $S$ is the antipode of $\mathrm{U}_{q}\left(\mathfrak{s l}_{2}\right)$.

The following maps are clearly $\mathrm{U}_{q}\left(\mathfrak{s l}_{2}\right)$-morphisms

$$
\begin{gathered}
\check{C}: \mathcal{K}_{0} \longrightarrow V \otimes V, \quad 1 \mapsto c_{0}, \\
\hat{C}: V \otimes V \longrightarrow \mathcal{K}_{0}, \quad v \otimes w \mapsto(v, w),
\end{gathered}
$$

as are the maps $\eta, \zeta: V \longrightarrow V$ respectively defined by the compositions

$$
\begin{aligned}
& \eta: V \stackrel{\sim}{\longrightarrow} \mathcal{K}_{0} \otimes V \stackrel{\check{C} \otimes \mathrm{id}}{\longrightarrow} V \otimes V \otimes V \stackrel{\mathrm{id} \otimes \hat{C}}{\longrightarrow} V, \\
& \zeta: V \stackrel{\sim}{\longrightarrow} V \otimes \mathcal{K}_{0} \stackrel{\mathrm{id} \otimes \check{C}}{\longrightarrow} V \otimes V \otimes V \stackrel{\hat{C} \otimes \mathrm{id}}{\longrightarrow} V .
\end{aligned}
$$

The statements in Lemma 4.3 are all either well-known or easily checked.

Lemma 4.3. Let $e=\check{C} \circ \hat{C}$. The following relations hold.

$$
\begin{array}{r}
\hat{C}(\check{C})=-\left(q+q^{-1}\right), \\
\eta=\zeta=\operatorname{id}_{V}, \\
e^{2}=-\left(q+q^{-1}\right) e .
\end{array}
$$

4.1.2. The universal $R$-matrix. As the universal $R$-matrix of $\mathrm{U}_{q}\left(\mathfrak{s l}_{2}\right)$ will play an important role in our development, we give some explicit information concerning it. Following [18, we define a functorial linear operator $\Xi$ as follows. For any pair of modules $M_{1}, M_{2}$ in $\mathcal{O}_{\text {int }}$, and weight vectors $w_{1} \in M_{1}$ and $w_{2} \in M_{2}$ with weights $k_{1}, k_{2}$ respectively,

$$
\Xi_{M_{1}, M_{2}}: M_{1} \otimes M_{2} \longrightarrow M_{1} \otimes M_{2}, \quad w_{1} \otimes w_{2} \mapsto q^{\frac{k_{1} k_{2}}{2}} w_{1} \otimes w_{2} .
$$

The universal $R$-matrix is the functorial linear isomorphism

$$
R=\Xi\left(\sum_{j=0}^{\infty} \frac{\left(q-q^{-1}\right)^{j}}{\llbracket j \rrbracket_{q} !} E^{j} \otimes F^{j}\right),
$$


where $\llbracket j \rrbracket_{q} !=\prod_{k=0}^{j} \llbracket k \rrbracket_{q}$ with $\llbracket k \rrbracket_{q}=\frac{1-q^{-2 k}}{1-q^{-2}}$. [Warning: this is not the usual definition of $q$-numbers.] For $i=1,2$, denote the representation of $\mathrm{U}_{q}\left(\mathfrak{s l}_{2}\right)$ on $M_{i}$ by $\pi_{i}$. Then the universal $R$-matrix acts on $M_{1} \otimes M_{2}$ by

$$
R_{M_{1}, M_{2}}=\Xi\left(\sum_{j=0}^{\infty} \frac{\left(q-q^{-1}\right)^{j}}{\llbracket j \rrbracket_{q} !} \pi_{1}\left(E^{j}\right) \otimes \pi_{2}\left(F^{j}\right)\right) .
$$

This is well defined, since $E$ and $F$ act locally nilpotently. The universal $R$-matrix has the following properties.

$$
\begin{gathered}
R_{M_{1}, M_{2}}\left(\pi_{1} \otimes \pi_{2}\right) \Delta(x)=\left(\pi_{1} \otimes \pi_{2}\right) \Delta^{\prime}(x) R_{M_{1}, M_{2}}, \quad \forall x \in \mathrm{U}_{q}\left(\mathfrak{s l}_{2}\right) \\
R_{M_{1} \otimes M_{2}, M_{3}}=R_{M_{1}, M_{3}} R_{M_{2}, M_{3}}, \quad R_{M_{1}, M_{2} \otimes M_{3}}=R_{M_{1}, M_{3}} R_{M_{1}, M_{2}}, \\
R_{M_{1}, M_{2}} R_{M_{1}, M_{3}} R_{M_{2}, M_{3}}=R_{M_{2}, M_{3}} R_{M_{1}, M_{3}} R_{M_{1}, M_{2}},
\end{gathered}
$$

where the last two equations are equalities of automorphisms of $M_{1} \otimes M_{2} \otimes M_{3}$. The last equation is the celebrated Yang-Baxter equation.

Let $P_{M_{1}, M_{2}}: M_{1} \otimes M_{2} \longrightarrow M_{2} \otimes M_{1}$ be the permutation $w \otimes w^{\prime} \mapsto w^{\prime} \otimes w$, and denote $\check{R}_{M_{1}, M_{2}}=P_{M_{1}, M_{2}} R_{M_{1}, M_{2}}: M_{1} \otimes M_{2} \longrightarrow M_{2} \otimes M_{1}$. Then

$$
\check{R}_{M_{1}, M_{2}}\left(\pi_{1} \otimes \pi_{2}\right) \Delta(x)-\left(\pi_{2} \otimes \pi_{1}\right) \Delta(x) \check{R}_{M_{1}, M_{2}}=0, \quad \forall x \in \mathrm{U}_{q}\left(\mathfrak{s l}_{2}\right),
$$

and the Yang-Baxter equation becomes the following "braid relation" among isomorphisms $M_{1} \otimes M_{2} \otimes M_{3} \longrightarrow M_{3} \otimes M_{2} \otimes M_{1}$ in $\mathcal{O}_{\text {int }}$.

$$
\begin{aligned}
& \left(\check{R}_{M_{2}, M_{3}} \otimes \operatorname{id}_{M_{1}}\right)\left(\operatorname{id}_{M_{2}} \otimes \check{R}_{M_{1}, M_{3}}\right)\left(\check{R}_{M_{1}, M_{2}} \otimes \operatorname{id}_{M_{3}}\right) \\
= & \left(\operatorname{id}_{M_{3}} \otimes \check{R}_{M_{1}, M_{2}}\right)\left(\check{R}_{M_{1}, M_{3}} \otimes \operatorname{id}_{M_{2}}\right)\left(\operatorname{id}_{M_{1}} \otimes \check{R}_{M_{2}, M_{3}}\right) .
\end{aligned}
$$

For $M_{1}=M_{2}=V(1)$, by looking at the action of $q^{\frac{1}{2}} \check{R}_{V, V}$ on the respective highest weight vectors of the simple submodules of $V \otimes V=V(2) \oplus V(0)$, it becomes evident that $q^{\frac{1}{2}} \check{R}_{V, V}$ has eigenvalues $q$ and $-q^{-1}$ on $V(2)$ and $V(0)$ respectively. Bearing in mind that $\check{C} \circ \hat{C}=-\left(q+q^{-1}\right)$ times the projection to $V(0)$ this may be restated as follows.

Lemma 4.4. The R-matrix $\check{R}_{V, V}$ satisfies the following relation.

$$
q^{\frac{1}{2}} \check{R}_{V, V}=q+\check{C} \circ \hat{C},
$$

where $\check{C}$ and $\hat{C}$ are as defined in (4.1).

Now let

$$
R^{T}=\Xi\left(\sum_{j=0}^{\infty} \frac{\left(q-q^{-1}\right)^{j}}{\llbracket j \rrbracket_{q} !} F^{j} \otimes E^{j}\right),
$$

and denote $R_{M_{1}, M_{2}}^{T}=R^{T}: M_{1} \otimes M_{2} \longrightarrow M_{1} \otimes M_{2}$. Then

$$
R_{M_{1}, M_{2}}^{T}=P_{M_{2}, M_{1}} R_{M_{2}, M_{1}} P_{M_{1}, M_{2}}: M_{1} \otimes M_{2} \longrightarrow M_{1} \otimes M_{2} .
$$

Furthermore, $\check{R}_{M_{2}, M_{1}} \check{R}_{M_{1}, M_{2}}=R_{M_{1}, M_{2}}^{T} R_{M_{1}, M_{2}}$, and

$$
R_{M_{1}, M_{2}}^{T} R_{M_{1}, M_{2}}=\Delta\left(v^{-1}\right)(v \otimes v): M_{1} \otimes M_{2} \longrightarrow M_{1} \otimes M_{2},
$$

where $v$ is Drinfeld's central element of $\mathrm{U}_{q}\left(\mathfrak{s l}_{2}\right)$ (see [18]). The element $v$ acts on any highest weight module with highest weight $\ell$ (i.e., a module which is generated by a highest weight vector of weight $\ell$ ) in $\mathcal{O}_{\text {int }}$ as multiplication by the scalar $q^{-\frac{1}{2} \ell(\ell+2)}$. 
Assume that $M_{1}$ and $M_{2}$ are both highest weight modules with highest weights $\ell_{1}$ and $\ell_{2}$ respectively. Then $R_{M_{1}, M_{2}}^{T} R_{M_{1}, M_{2}}$ acts on a highest weight submodule $M^{\prime}$ of $M_{1} \otimes M_{2}$ with highest weight $\ell$ as

$$
\begin{aligned}
& \left.R_{M_{1}, M_{2}}^{T} R_{M_{1}, M_{2}}\right|_{M^{\prime}}=q^{\chi\left(\ell, \ell_{1}, \ell_{2}\right)} \mathrm{id}_{M^{\prime}}, \text { where } \\
& \chi\left(\ell, \ell_{1}, \ell_{2}\right)=\frac{\ell(\ell+2)}{2}-\frac{\ell_{1}\left(\ell_{1}+2\right)}{2}-\frac{\ell_{2}\left(\ell_{2}+2\right)}{2} .
\end{aligned}
$$

If $m_{+}$is the highest weight vector of $M_{1}$, and $v \in M_{2}$ is a vector of weight $j$, we have $K m_{+}=q^{\ell_{1}} m_{+}$and $K v=q^{j} v$. Then

$$
R_{M_{1}, M_{2}}^{T} R_{M_{1}, M_{2}}\left(m_{+} \otimes v\right)=\sum_{k=0}^{\infty} \frac{\left(q-q^{-1}\right)^{k} q^{j \ell_{1}+k\left(\ell_{1}-j-2 k\right)}}{\llbracket k \rrbracket_{q} !} F^{k} m_{+} \otimes E^{k} v
$$

4.2. A tensor functor. We again fix $V=V(1)$, and for any integer $\ell$, let $M$ be either $M(\ell)$ or $V(\ell)$. We shall adopt the following notation. Recall that $\mathcal{C}=\{m, v\}$; for any sequence $A=\left(a_{1}, a_{2}, \ldots, a_{r}\right)$ with $a_{j} \in \mathcal{C}$, write

$$
U^{A}=U^{a_{1}} \otimes U^{a_{2}} \otimes \cdots \otimes U^{a_{r}},
$$

where $U^{m}=M$ and $U^{v}=V$. We set $U^{\varnothing}=\mathcal{K}_{0}$ for the empty sequence $\varnothing$.

Recall that there exists a canonical tensor functor from the category of directed coloured ribbon graphs to the category of finite dimensional representations of any quantum group; see [24, Theorem 5.1]. Adapting that functor to our context yields the following result.

Theorem 4.5. Let $V=V(1)$, and for any integer $\ell$ let $M$ be either $M(\ell)$ or $V(\ell)$. There exists a unique covariant linear functor $\hat{\mathcal{F}}: \mathcal{R} \mathcal{T} \longrightarrow \mathcal{O}_{\text {int }}$, which satisfies the following properties.

(1) The functor respects the tensor products of $\mathcal{R} \mathcal{T}$ and $\mathcal{O}_{\text {int }}$;

(2) $\hat{\mathcal{F}}$ sends the object $A$ of $\mathcal{R} \mathcal{T}$ to $\hat{\mathcal{F}}(A)=U^{A}$; and

(3) $\hat{\mathcal{F}}$ maps the generators of the morphism spaces as indicated below.
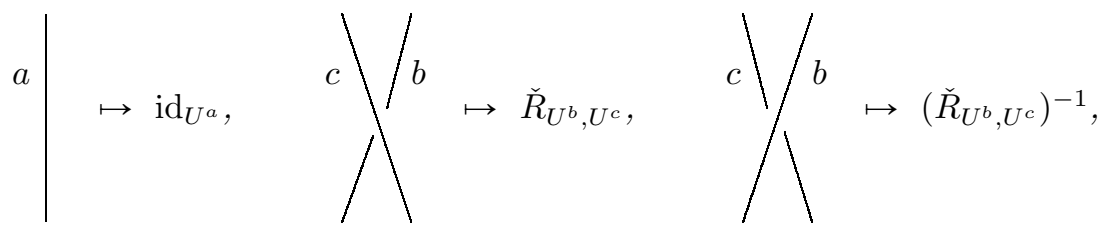

for all $a, b, c \in \mathcal{C}$ with $b, c$ not both equal to $m$, and

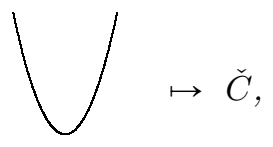

$v$

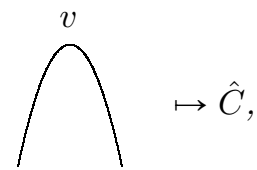

$\rightarrow \hat{C}$

where the maps $\check{C}: \mathcal{K}_{0} \longrightarrow V \otimes V$ and $\hat{C}: V \otimes V \longrightarrow \mathcal{K}_{0}$ are defined by (4.1) and (4.2) respectively. 
Proof. Suppose for the moment that the functor $\widehat{\mathcal{F}}$ as defined in (2) and (3) is well defined on morphisms, that is, that

The relations in part (3) of Theorem 3.1 are preserved by $\hat{\mathcal{F}}$.

Then equation (4.17) and property (3) of the statement, which defines the images of the generators of morphisms under $\hat{\mathcal{F}}$, define the functor uniquely. The functor evidently respects the tensor products for objects, since for any objects $A$ and $B$ in $\mathcal{R} \mathcal{T}$, we have

$$
\widehat{\mathcal{F}}(A \otimes B)=U^{(A, B)}=U^{A} \otimes U^{B} .
$$

The statement (1) now reduces to showing that $\hat{\mathcal{F}}$ respect tensor products for morphisms, that is, for any two morphisms $D, D^{\prime}$ in $\mathcal{R} \mathcal{T}$,

$$
\widehat{\mathcal{F}}\left(D \otimes D^{\prime}\right)=\widehat{\mathcal{F}}(D) \otimes \widehat{\mathcal{F}}\left(D^{\prime}\right),
$$

which will follow from (4.16). Thus we are reduced to proving the latter statement.

Clearly $\hat{\mathcal{F}}$ preserves relations (a). It also preserves relations (b) since the $R$ matrices satisfy the Yang-Baxter equation. It follows from (4.4) that the straightening relations (c) are also preserved.

To prove the sliding relations, let us write

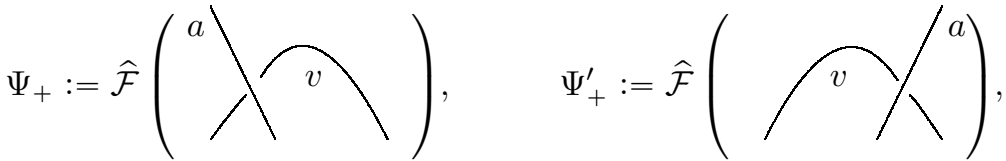

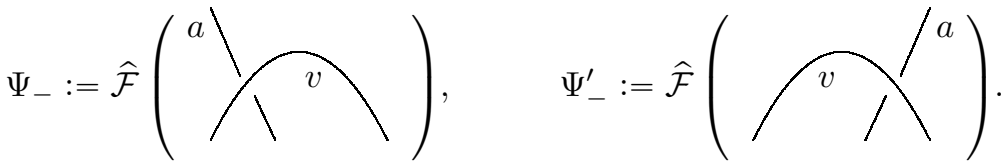

Let $v, v^{\prime} \in V$ be vectors with weights $\ell$ and $\ell^{\prime}$ respectively, and let $w \in U^{a}$ be a vector with weight $k$. Then we have

$$
\begin{aligned}
& \Psi_{+}\left(v \otimes w \otimes v^{\prime}\right)=q^{\frac{1}{2} k \ell}\left(v, v^{\prime}\right) w+\left(q-q^{-1}\right) q^{\frac{1}{2}(k-2)(\ell+2)}\left(E v, v^{\prime}\right) F w, \\
& \Psi_{+}^{\prime}\left(v \otimes w \otimes v^{\prime}\right)=q^{-\frac{1}{2} k \ell^{\prime}}\left(v, v^{\prime}\right) w-\left(q-q^{-1}\right) q^{-\frac{1}{2} k \ell^{\prime}}\left(v, E v^{\prime}\right) F w .
\end{aligned}
$$

Invariance of the bilinear form implies that $\left(v, E v^{\prime}\right)=-\left(E v, K v^{\prime}\right)=-q^{\ell^{\prime}}\left(E v, v^{\prime}\right)$. Also note that $\left(v, v^{\prime}\right)=0$ unless $\ell+\ell^{\prime}=0$, and $\left(E v, v^{\prime}\right)=\left(v, E v^{\prime}\right)=0$ unless $\ell=\ell^{\prime}=-1$. Hence

$$
\Psi_{+}\left(v \otimes w \otimes v^{\prime}\right)=q^{\frac{1}{2} k \ell}\left(v, v^{\prime}\right) w+\left(q-q^{-1}\right) q^{\frac{1}{2}(k-2)}\left(E v, v^{\prime}\right) F w=\Psi_{+}^{\prime}\left(v \otimes w \otimes v^{\prime}\right) .
$$

Similarly we have

$$
\begin{aligned}
& \Psi_{-}\left(v \otimes w \otimes v^{\prime}\right)=q^{-\frac{1}{2} k \ell}\left(v, v^{\prime}\right) w-\left(q-q^{-1}\right) q^{-\frac{1}{2} k \ell}\left(F v, v^{\prime}\right) E w, \\
& \Psi_{-}^{\prime}\left(v \otimes w \otimes v^{\prime}\right)=q^{\frac{1}{2} k \ell^{\prime}}\left(v, v^{\prime}\right) w+\left(q-q^{-1}\right) q^{\frac{1}{2}(k+2)\left(\ell^{\prime}-2\right)}\left(v, F v^{\prime}\right) E w .
\end{aligned}
$$

In this case, $\left(F v, v^{\prime}\right)=-\left(v, K F v^{\prime}\right)=-q^{-1}\left(v, F v^{\prime}\right)$, and $\left(F v, v^{\prime}\right)=\left(v, F v^{\prime}\right)=0$ unless $\ell=\ell^{\prime}=1$. We still have $\left(v, v^{\prime}\right)=0$ unless $\ell+\ell^{\prime}=0$. Hence

$\Psi_{-}\left(v \otimes w \otimes v^{\prime}\right)=q^{-\frac{1}{2} k \ell}\left(v, v^{\prime}\right) w+\left(q-q^{-1}\right) q^{-\frac{1}{2}(k+2)}\left(v, F v^{\prime}\right) E w=\Psi_{-}^{\prime}\left(v \otimes w \otimes v^{\prime}\right)$. 
Now consider the twists. Let
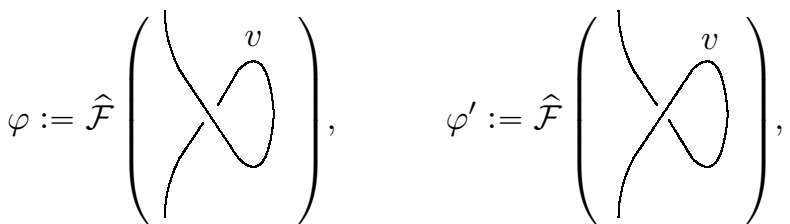

which are scalar multiples of $\operatorname{id}_{V}$. By direct computations, one can verify that

$$
\varphi v_{1}=-q^{\frac{3}{2}} v_{1}, \quad \varphi^{\prime} v_{-1}=-q^{-\frac{3}{2}} v_{-1} .
$$

Hence $\varphi=-q^{\frac{3}{2}} \operatorname{id}_{V}, \varphi^{\prime}=-q^{-\frac{3}{2}} \mathrm{id}_{V}$, and $\varphi \circ \varphi^{\prime}=\mathrm{id}_{V}$. Another way to compute this is to use the well known relationship between the $R$-matrix and Drinfeld's central element. By taking into account the $q$-skew nature of the bilinear form, we immediately obtain

$$
\varphi=-q^{\frac{3}{2}} \mathrm{id}_{V}, \quad \varphi^{\prime}=-q^{-\frac{3}{2}} \mathrm{id}_{V} .
$$

This completes the proof of Theorem 4.5

Remark 4.6. Note that when constructing representations of the Artin braid group, one has the freedom of multiplying the $R$-matrices by an invertible scalar. However the sliding relations remove this freedom in the definition of the functor $\hat{\mathcal{F}}$ (cf. Remark 3.8).

Theorem 4.7. The functor $\hat{\mathcal{F}}: \mathcal{R T} \longrightarrow \mathcal{O}_{\text {int }}$ of Theorem 4.5 factors through $\mathcal{M T} \mathcal{L}\left(q, q^{\ell+1}\right)$.

Proof. It follows from the property of $\check{R}$ given in Lemma 4.4 that the functor $\hat{\mathcal{F}}$ respects the first two skein relations of $\mathcal{M T} \mathcal{L}(q, \Omega)$. By Lemma 3.4 it therefore also respects the free loop removal relation (cf. also (4.3)). We now set

$$
\Omega=q^{\ell+1} \text {. }
$$

We want to show that $\hat{\mathcal{F}}$ preserves the third skein relation of $\mathcal{M T} \mathcal{L}(q, \Omega)$ (see Lemma 3.15). We have

$$
\xi:=\hat{\mathcal{F}}
$$

In the case $M=M(\ell)$, if $\ell \neq-1$, we have $M \otimes V=M(\ell+1) \oplus M(\ell-1)$. Hence $\xi=\check{R}_{V, M} \check{R}_{M, V}$ has two eigenvalues $q^{\chi_{ \pm}}$, which we can compute by using (4.14) to obtain

$$
\chi_{ \pm}=\frac{1}{2}(\ell \pm 1)(\ell+2 \pm 1)-\frac{1}{2} \ell(\ell+2)-\frac{3}{2}= \pm(\ell+1)-1 .
$$

Hence $\xi$ satisfies the quadratic relation

$$
(q \xi-\Omega)\left(q \xi-\Omega^{-1}\right)=0 .
$$

If $\ell=-1$, we do not have such a decomposition for $M \otimes V$. However, we can directly verify the skein relation. Since $\check{R}_{V, M} \check{R}_{M, V}$ is a $\mathrm{U}_{q}\left(\mathfrak{s l}_{2}\right)$-morphism, we only need to verify this for the two vectors $m_{+} \otimes v_{1}$ and $m_{+} \otimes v_{-1}$, as they generate 
$M \otimes V$. It is clear that $m_{+} \otimes v_{1}$ is an eigenvector of $R_{M, V}^{T} R_{M, V}$ with eigenvalue $q^{-1}$. For the vector $m_{+} \otimes v_{-1}$, we use (4.7) and (4.13) to obtain the following relations.

$$
\begin{aligned}
R_{M, V}^{T} R_{M, V}\left(m_{+} \otimes v_{-1}\right) & =q m_{+} \otimes v_{-1}+q^{-1}\left(q-q^{-1}\right) F m_{+} \otimes v_{1}, \\
\left(R_{M, V}^{T} R_{M, V}\right)^{2}\left(m_{+} \otimes v_{-1}\right) & =\left(2-q^{-2}\right) m_{+} \otimes v_{-1}+2 q^{-2}\left(q-q^{-1}\right) F m_{+} \otimes v_{1} .
\end{aligned}
$$

Combining these we arrive at $\left(\check{R}_{V, M} \check{R}_{M, V}-q^{-1}\right)^{2}\left(m_{+} \otimes v_{-1}\right)=0$. Hence we have proved that in this case

$$
(q \xi-1)^{2}=0 .
$$

For $M=V(\ell)$, we only need to consider $\ell \geqslant 0$, since $V(\ell)=M(\ell)$ if $\ell<0$. We have $V(\ell) \otimes V=V(\ell+1) \oplus V(\ell-1)$ for $\ell>0$, and $V(0) \otimes V=V$. It follows from these decompositions that the relation (4.19) is also satisfied in this case.

This proves that $\hat{\mathcal{F}}$ preserves the third skein relation for $\mathcal{M T} \mathcal{L}\left(q, q^{\ell+1}\right)$.

We now verify the tangled loop removal relation for both $M(\ell)$ and $V(\ell)$. We note that for any linear transformation $\phi$ of $V$,

$$
\hat{C}\left(\phi \otimes \operatorname{id}_{V}\right) \check{C}(1)=-q\left(\phi v_{1}, v_{-1}\right)+\left(\phi v_{-1}, v_{1}\right)=-\operatorname{tr}_{V}(K \phi) .
$$

Hence

$$
\Phi:=\hat{\mathcal{F}}
$$

It follows from [29, Proposition 1] that $\Phi$ is a scalar multiple of $\mathrm{id}_{M}$. To compute the scalar, we consider the action of $\Phi$ on the highest weight vector $m_{+}$of $M$. Using (4.15), we obtain

$$
\Phi m_{+}=-\operatorname{tr}\left(\left(\begin{array}{cc}
q & 0 \\
0 & q^{-1}
\end{array}\right)\left(\begin{array}{cc}
q^{\ell} & q^{-1}\left(q-q^{-1}\right) \\
0 & q^{-\ell}
\end{array}\right)\right) m_{+}=-\left(\Omega+\Omega^{-1}\right) m_{+} .
$$

This leads to $\Phi=-\left(\Omega+\Omega^{-1}\right) \operatorname{id}_{M}$, and hence $\hat{\mathcal{F}}$ respects the tangled loop removal relation.

This completes the proof of Theorem 4.7.

4.3. An equivalence of categories. In this section, we take $V=V(1)$ and $M=M(\ell)$ for $\ell \geqslant-1$. Let $\mathcal{T}$ be the full subcategory of the category $\mathcal{O}_{\text {int }}$ of $\mathrm{U}_{q}\left(\mathfrak{s l}_{2}\right)$-modules with objects $M \otimes V^{\otimes r}$ for $r=0,1, \ldots$ Regard $\mathcal{T} \mathcal{L B}\left(q, q^{\ell+1}\right)$, the Temperley-Lieb category of type $B$, as a full subcategory of $\mathcal{M T} \mathcal{L}\left(q, q^{\ell+1}\right)$.

Theorem 4.5 enables us to define the following functors.

Definition 4.8. Let $\mathcal{F}: \mathcal{M T} \mathcal{L}\left(q, q^{\ell+1}\right) \longrightarrow \mathcal{O}_{\text {int }}$ be the functor defined by the commutative diagram

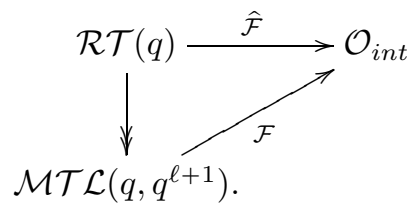

It is clear that $\mathcal{F}$ sends objects and morphisms in $\mathcal{T} \mathcal{L B}\left(q, q^{\ell+1}\right)$ to $\mathcal{T}$. Hence the restriction of the functor $\mathcal{F}$ to $\mathcal{T} \mathcal{L B}\left(q, q^{\ell+1}\right)$ leads to a covariant functor

$$
\mathcal{F}^{\prime}: \mathcal{T} \mathcal{L} \mathcal{B}\left(q, q^{\ell+1}\right) \longrightarrow \mathcal{T} \text {. }
$$


Theorem 4.9. Let $V=V(1)$ and $M=M(\ell)$. Then for all $\ell \geqslant-1$, the functor $\mathcal{F}^{\prime}$ : $\mathcal{T} \mathcal{L B}\left(q, q^{\ell+1}\right) \longrightarrow \mathcal{T}$ is an equivalence of categories, where $\mathcal{T}$ is the full subcategory of the category $\mathcal{O}_{\text {int }}$ with objects $M \otimes V^{\otimes r}$ for $r=0,1, \ldots$

Proof. We will prove the equivalence of categories by showing that the functor $\mathcal{F}^{\prime}$ is essentially surjective and fully faithful.

We set $\Omega=q^{\ell+1}$ in this proof.

The essential surjectivity is clear, since $\mathcal{F}^{\prime}\left(m, v^{r}\right)=M \otimes V^{\otimes r}$ for all $r$.

Since $V$ is self dual, $\operatorname{Hom}_{\mathcal{T}}\left(M \otimes V^{\otimes r}, M \otimes V^{\otimes s}\right) \cong \operatorname{Hom}_{\mathcal{T}}\left(M, M \otimes V^{\otimes(r+s)}\right)$ as vector spaces for all $r$ and $s$. In view of Lemma 3.19, we have $\operatorname{Hom}_{\mathcal{T} \mathcal{L B}(q, \Omega)}\left(\left(m, v^{r}\right)\right.$, $\left.\left(m, v^{s}\right)\right) \cong \operatorname{Hom}_{\mathcal{T} \mathcal{L B}(q, \Omega)}\left(m,\left(m, v^{r+s}\right)\right)$. Hence in order to prove that the functor $\mathcal{F}^{\prime}$ is fully faithful, it suffices to show that $\mathcal{F}^{\prime}$ defines isomorphisms

$$
\operatorname{Hom}_{\mathcal{T} \mathcal{L B}(q, \Omega)}\left(m,\left(m, v^{r}\right)\right) \stackrel{\sim}{\longrightarrow} \operatorname{Hom}_{\mathcal{T}}\left(M, M \otimes V^{r}\right)
$$

for all $r$. We shall do this by showing that for all $r$,

$$
\begin{gathered}
\operatorname{dim} \operatorname{Hom}_{\mathcal{T}}\left(M, M \otimes V^{\otimes r}\right)=\operatorname{dim} \operatorname{Hom}_{\mathcal{T} \mathcal{L B}(q, \Omega)}\left(m,\left(m, v^{r}\right)\right), \text { and } \\
\operatorname{dim} \mathcal{F}^{\prime}\left(\operatorname{Hom}_{\mathcal{T} \mathcal{L B}(q, \Omega)}\left(m,\left(m, v^{r}\right)\right)\right)=\operatorname{dim} \operatorname{Hom}_{\mathcal{T} \mathcal{L B}(q, \Omega)}\left(m,\left(m, v^{r}\right)\right) .
\end{gathered}
$$

Consider first $\operatorname{Hom}_{\mathcal{T}}\left(M, M \otimes V^{\otimes r}\right)$. We decompose $V^{\otimes r}$ with respect to the joint action of $\mathrm{U}_{q}\left(\mathfrak{s l}_{2}\right)$ and $\mathrm{TL}_{r}(q)$ to obtain $V^{\otimes r}=\oplus_{t} V(t) \otimes W_{t}(r)$, where the direct sum is over all $t$ such that $0 \leqslant t \leqslant r$ and $r-t$ is even. Since $\ell \geqslant-1$, we can apply part (2) of Lemma 4.2 to obtain

$$
\begin{aligned}
\operatorname{Hom}_{\mathrm{U}_{q}\left(\mathfrak{s l}_{2}\right)}\left(M, M \otimes V^{\otimes r}\right) & =\bigoplus_{t} \operatorname{Hom}_{\mathrm{U}_{q}\left(\mathfrak{s l}_{2}\right)}(M, M \otimes V(t)) \otimes W_{t}(r) \\
& =\bigoplus_{t} V(t)_{0} \otimes W_{t}(r) .
\end{aligned}
$$

If $r$ is odd, then all $t$ are odd, hence all $V(t)_{0}=0$. If $r=2 N$ is even,

$$
\operatorname{Hom}_{\mathrm{U}_{q}\left(\mathfrak{s} \mathfrak{s}_{2}\right)}\left(M, M \otimes V^{\otimes 2 N}\right)=\bigoplus_{t=0}^{N} V(2 t)_{0} \otimes W_{2 t}(2 N)=\bigoplus_{t=0}^{N} W_{2 t}(2 N) .
$$

In view of Lemma 3.24 and its proof, this establishes equation (4.20).

Next we consider $\mathcal{F}^{\prime}\left(\operatorname{Hom}_{\mathcal{T} \mathcal{L B}(q, \Omega)}\left(m,\left(m, v^{r}\right)\right)\right)$.

Applying $\mathcal{F}^{\prime}$ to the filtration $(\underline{3.10})$ of $W(2 N)$ by $\mathrm{TL}_{2 N}(q)$-modules $F_{t} W(2 N)$, and writing $\mathcal{F}_{i} W(2 N)=\mathcal{F}^{\prime}\left(F_{i} W(2 N)\right)$, we obtain

$$
\mathcal{F}_{N} W(2 N) \supset \mathcal{F}_{N-1} W(2 N) \supset \cdots \supset \mathcal{F}_{1} W(2 N) \supset \mathcal{F}_{0} W(2 N) \supset \varnothing .
$$

This is a filtration of modules for $\mathcal{F}^{\prime}\left(\operatorname{End}^{0}(2 N)\right)$, which by [19, Thm. 3.5] is isomorphic to $\mathrm{TL}_{r}(2 N)$. For any $i$, if the quotient $W_{2 i}^{\prime}(2 N):=\frac{\mathcal{F}_{i} W(2 N)}{\mathcal{F}_{i-1} W(2 N)} \neq 0$, then it must be isomorphic to the cell module $W_{2 i}(2 N)$ as $\mathrm{TL}_{r}(2 N)$-module. Therefore, if we can show that $W_{2 i}^{\prime}(2 N) \neq 0$ for all $i$, then (4.21) follows in view of Lemma 3.24

Assume to the contrary that $W_{2 i}^{\prime}(2 N)=0$ for some $i$. This happens precisely if, given any distinguished diagram $D \in F_{i} W(2 N)$ with $i$ thin arcs entangled with the pole, there is an element $D^{r e d} \in F_{i-1} W(2 N)$ such that

$$
\mathcal{F}^{\prime}(D)-\mathcal{F}^{\prime}\left(D^{r e d}\right)=0 .
$$

Let $D$ be given by the distinguished diagram in Figure 12, We shall show that $\mathcal{F}^{\prime}(D) \notin \mathcal{F}^{\prime}\left(F_{i-1} W(2 N)\right)$. 


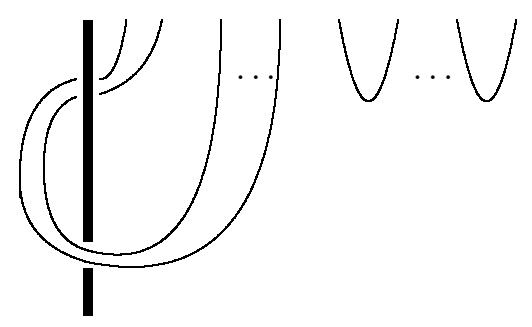

Figure 12. Diagram $D: m \rightarrow\left(m, v^{2 N}\right)$

Take the morphisms $A:\left(m, v^{2 N}\right) \rightarrow\left(m, v^{2 i}\right), I_{i}: v^{i} \rightarrow v^{i}$ and $S:\left(m, v^{3 i}\right) \rightarrow$ $\left(m, v^{i}\right)$, which are respectively given by

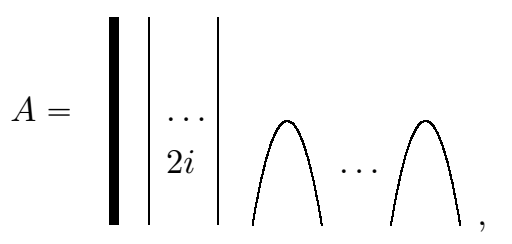

$$
I_{i}=\left|\begin{array}{c} 
\\
\cdots \\
i
\end{array}\right|,
$$

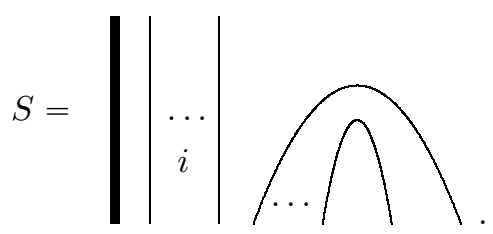

We first compose $D$ and the related $D^{r e d}$ with $A$ to obtain $A D$ and $A D^{r e d}$, then tensor them with $I_{i}$ to obtain $A D \otimes I_{i}$ and $A D^{\text {red }} \otimes I_{i}$, and finally compose these morphisms with $S$ to obtain $S\left(A D \otimes I_{i}\right)$ and $S\left(A D^{\text {red }} \otimes I_{i}\right)$. Write $\tilde{D}=$ $\delta^{-N+i} S\left(A D \otimes I_{i}\right)$ and $\tilde{D}^{r e d}=\delta^{-N+i} S\left(A D^{r e d} \otimes I_{i}\right)$; these are both endomorphisms of $\left(m, v^{i}\right)$.

Now if $W_{2 i}^{\prime}(2 N)=0$ for some $i$, equation (4.23) implies

$$
\mathcal{F}^{\prime}(\tilde{D})-\mathcal{F}^{\prime}\left(\tilde{D}^{r e d}\right)=0
$$

The diagram of $\tilde{D}$ is given by Figure 13. The morphism $\tilde{D}^{\text {red }}$ is spanned by diagrams with less than $i$ thin arcs tangled with the thick arc.

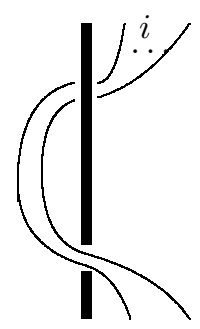

Figure 13. Diagram $\tilde{D}:\left(m, v^{i}\right) \rightarrow\left(m, v^{i}\right)$

Let $\mathbf{w}=m_{+} \otimes v_{-1}^{\otimes i}$, where $v_{-1}^{\otimes i}=\underbrace{v_{-1} \otimes v_{-1} \otimes \cdots \otimes v_{-1}}_{i}$ and let us compare $\mathcal{F}^{\prime}(\tilde{D})(\mathbf{w})$ and $\mathcal{F}^{\prime}\left(\tilde{D}^{r e d}\right)(\mathbf{w})$. By (4.15), we have

$$
\mathcal{F}^{\prime}(\tilde{D})(\mathbf{w})=\sum_{k=0}^{i} \frac{\left(q-q^{-1}\right)^{k} q^{-i \ell+k(\ell+i-2 k)}}{\llbracket k \rrbracket_{q} !} F^{k} m_{+} \otimes E^{k} v_{-1}^{\otimes i}
$$


In particular, the vector $F^{i} m_{+} \otimes v_{1}^{\otimes i}$ appears in $\mathcal{F}^{\prime}(\tilde{D})(\mathbf{w})$ with a nonzero coefficient. This vector is nonzero since $F^{i} m_{+} \neq 0$ for all $i$ in the Verma module $M$.

Turning to $\mathcal{F}^{\prime}\left(\tilde{D}^{r e d}\right)(\mathbf{w})$, we note that $\mathbf{w}=m_{+} \otimes v_{-1}^{\otimes i}$ is annihilated by the images under $\mathcal{F}^{\prime}$ of all diagrams from $\left(m, v^{i}\right)$ to $\left(m, v^{i-2}\right)$ which have an arc $U$ as depicted below, since the invariant form on $V(1)$ satisfies $\left(v_{-1}, v_{-1}\right)=0$.

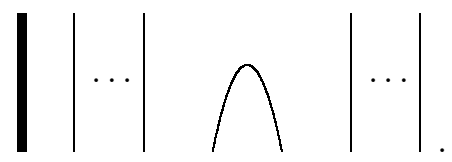

Thus among all the diagrams in $\tilde{D}^{\text {red }}$, only those shown in Figure 14 with $t<i$ could have nonzero contributions to $\mathcal{F}^{\prime}\left(\tilde{D}^{r e d}\right)(\mathbf{w})$. Using (4.15), we obtain

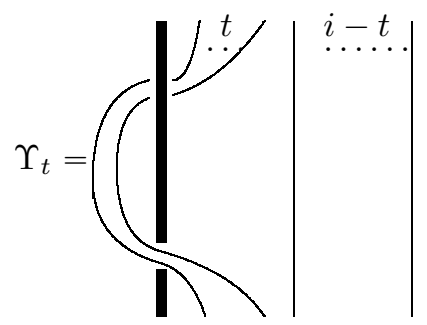

FiguRE 14. A diagram in $\tilde{D}^{\text {red }}$

$$
\mathcal{F}^{\prime}\left(\Upsilon_{t}\right)(\mathbf{w})=\sum_{k=0}^{t} \frac{\left(q-q^{-1}\right)^{k} q^{-t \ell+k(\ell+t-2 k)}}{\llbracket k \rrbracket_{q} !} F^{k} m_{+} \otimes E^{k} v_{-1}^{\otimes t} \otimes v_{-1}^{\otimes(i-t)} .
$$

Note that for all $t<i$, the vector $F^{i} m_{+} \otimes v_{1}^{\otimes i}$ never appears in $\mathcal{F}^{\prime}\left(\Upsilon_{t}\right)(\mathbf{w})$ with a non-zero coefficient. Thus (4.24) does not hold for any element $D^{r e d} \in F_{i-1} W(2 N)$. Hence $W_{2 i}^{\prime}(2 N)=W_{2 i}(2 N)$ for all $i$, and equation (4.21) is proved.

We have now shown that $\mathcal{F}^{\prime}$ is fully faithful, proving Theorem 4.9

4.4. Quantum Schur-Weyl duality. The algebra $\mathbb{T} \mathbb{B} \mathbb{B}_{r}(q, Q)$, defined in Section 2 as the algebra of endomorphisms of $r$ in the category $\mathbb{T} \mathbb{R}(q, Q)$, is generated [14, (5.7)] by the elements $c_{i}:=I^{\otimes(i-1)} \otimes(U \circ A) \otimes I^{\otimes(r-i-1)}(i=1,2, \ldots, r-1)$ and $c_{0}:=C_{0} \otimes I^{\otimes(r-1)}$, subject to the relations set out in [14, Prop. (5.3)]. Likewise, the endomorphism algebra $\operatorname{TLB}_{r}(q, \Omega)$ of the object $\left(m, v^{r}\right)$ in $\mathcal{T} \mathcal{L B}(q, \Omega)$ has generators $C_{i}, i=1, \ldots, r-1$, defined in analogy with the $c_{i}$ using the elements $I, A$ and $U$ of Section 3.5, as well as the element $L \otimes I^{\otimes(r-1)}$, which we refer to as $L \in \operatorname{TLB}_{r}(q, \Omega)$.

Corollary 4.10. For any integer $r>0$, there is an isomorphism of algebras

$$
\mathbb{T} \mathbb{B} \mathbb{B}_{r}(q, Q) \longrightarrow \operatorname{TLB}_{r}(q, \sqrt{-1} Q),
$$

which, in the notation explained above, takes the generators $c_{i}$ to $C_{i}(i=1, \ldots, r-1)$ and takes $c_{0}$ to $-\sqrt{-1} q L-Q I^{\otimes r}$, where $\sqrt{-1}$ is a fixed square root of -1 . 
It is evidently a consequence of Theorem 4.9 and Corollary 4.10 that:

Proposition 4.11. Let $V=V(1)$ and $M=M(\ell)$ with $\ell \geqslant-1$. Then for each $r=1,2, \ldots$, there are the following isomorphisms of associative algebras

$$
\operatorname{TLB}_{r}\left(q, q^{\ell+1}\right) \stackrel{\sim}{\longrightarrow} \operatorname{End}_{\mathrm{U}_{q}\left(\mathfrak{s l}_{2}\right)}\left(M \otimes V^{\otimes r}\right) \stackrel{\sim}{\longrightarrow} \mathbb{T} \llbracket \mathbb{B}_{r}\left(q, \sqrt{-1} q^{\ell+1}\right) .
$$

Remark 4.12. A similar Schur-Weyl duality between classical $\mathfrak{s l}_{n}$ and the degenerate affine Hecke algebra was established in [3]. Our main result is that when $n=2$ one can frame an equivalence, which may extend to the case where $q$ is a root of unity (cf. 2] for the classical case).

4.5. Semisimplicity of endomorphism algebras. It is apparent that analysis of the algebras $\mathbb{T} \mathbb{B} \mathbb{B}_{n}(q, Q)$ may be approached through their cellular structure outlined above. This makes it possible to analyse the representations $M(\ell) \otimes V(1)^{\otimes r}$. In this section we determine precisely when $\operatorname{End}_{\mathrm{U}_{q}\left(\mathfrak{s l}_{2}\right)}\left(M(\ell) \otimes V(1)^{\otimes r}\right)$ is semisimple. For ease of exposition, we assume throughout this section that $Q$ and $\Omega$ are both in the field $\mathcal{K}_{0}$ and we have chosen a fixed square root $\sqrt{-1} \in \mathcal{K}_{0}$.

4.5.1. Semisimplicity of $\mathbb{T} \mathbb{B} \mathbb{B}_{r}(q, \Omega)$. This may be approached as in 7 for positive characteristic. However here we shall use the approach of [13, 14 which relates $\mathbb{T} \mathbb{B} \mathbb{B}_{r}(q, Q)$ to the (unextended) affine Temperley-Lieb algebra $T_{r}^{a}(q)$ as in 14, as well as the complete analysis of its cell modules $W_{t, z}(r)$ given in [13. The results we quote as background may all be found in [14, $\S \S 6,10]$.

For each integer $r \geqslant 0$ define the following sets of parameters:

$$
\begin{aligned}
\Lambda(r) & =\{t \in \mathbb{Z} \mid 0 \leqslant t \leqslant r \text { and } r-t \in 2 \mathbb{Z}\} \\
\Lambda_{B}(r) & =\{t \in \mathbb{Z}|| t \mid \in \Lambda(r)\} \\
\Lambda^{a}(r) & =\left\{(t, z) \in \mathbb{Z} \times \mathcal{K}_{0}^{*} \mid t \in \Lambda(r)\right\} / \sim,
\end{aligned}
$$

where in the third line, we declare $(0, z) \sim\left(0, z^{-1}\right)$.

The sets $\Lambda(r)$ and $\Lambda_{B}(r)$ are posets, with $\Lambda(r)$ being ordered in the obvious way, $\Lambda_{B}(r)$ ordered according to $|t|$, with $|t| \geqslant t$. The set $\Lambda^{a}(r)$ indexes the cell modules of $T_{r}^{a}(q)$, among which all homomorphisms are known.

Any cellular algebra is semisimple if and only if there are no non-trivial homomorphisms among its cell modules $\left[12\right.$. The key to the semisimplicity of $\mathbb{\mathbb { L }} \mathbb{B}_{r}(q, Q)$ is therefore the following result. For the notation, the reader is referred to [14, §5].

Theorem 4.13 ([14, Cor (5.11), Thm. (6.15)]).

(1) For $1 \leqslant i \leqslant r-1$, let $t_{i}=c_{i}+q \in \mathbb{T} \mathbb{L} \mathbb{B}_{r}(q, Q)$, and let $t_{0}=c_{0}+Q$. There is a surjective homomorphism $g: T_{r}^{a}(q) \longrightarrow \mathbb{T} \mathbb{\mathbb { B } _ { r }}(q, Q)$ defined by $g\left(f_{i}\right)=c_{i}$ for $i=1, \ldots, r-1$, and $g(\tau)=\sqrt{-1} q^{\frac{1}{2}(n-2)} t_{0} t_{1} \ldots t_{r-1}$, where $\tau$ is the "twist" diagram in $T_{r}^{a}(q)$.

(2) Denoting by $g^{*}(M)$ the pullback to $T_{r}^{a}(q)$ of a $\mathbb{\square} \mathbb{B} \mathbb{B}_{r}(q, Q)$-module $M$, we have, for $t \in \Lambda_{B}(r)$,

$$
g^{*}\left(W_{t}(r)\right) \cong W_{|t|, z_{t}^{\varepsilon}}(r)
$$

where $\varepsilon_{t}=\frac{t}{|t|}= \pm 1$ and $z_{t}=(-1)^{t+\frac{1}{2}} q^{-\frac{t}{2}} Q^{-1}$.

Since all homomorphisms among the modules $W_{t, z}$ are known, Theorem4.13 may be used to determine whether $\mathbb{T} \mathbb{R} \mathbb{B}_{r}(q, Q)$ is semisimple, since $\mathbb{T} \mathbb{B}$-homomorphisms among the $W_{t}(r)$ are precisely $T^{a}$-homomorphisms among the lifts. We begin by 
explaining when we have a non-trivial homomorphism between two cell modules $W_{t, z}$.

Define a preorder on $\Lambda^{a}(r)$ as follows. Say that $(t, z) \prec(s, y)((t, z),(s, y) \in$ $\left.\Lambda^{a}(r)\right)$ if for some $\varepsilon= \pm 1$ we have

$$
s=t+2 m \text { for some } m>0, \quad y=q^{-\varepsilon m} z, \quad \text { and } \quad z^{2}=q^{\varepsilon s} .
$$

A short calculation using equation (4.26) reveals that there is a non-zero homomorphism of cell modules $W_{s}(r) \longrightarrow W_{t}(r)$ for $\mathbb{T} \mathbb{R} \mathbb{B}_{r}(q, Q)\left(t, s \in \Lambda_{B}(r)\right)$ if and only if either $W_{t}(r) \cong W_{-t}(r)$ (see Corollary 4.14) for some $t>0$ or if the following conditions hold:

(i) $\exists t, s \in \Lambda(r)$ such that $s=t+2 m>t \geqslant 0$, and $Q=\sqrt{-1} q^{-(t+m)}$;

(ii) $\exists t<0, s>0 \in \Lambda_{B}(r)$, such that $t=-2 m, s=4 m$ and $Q=\sqrt{-1} q^{m}$;

(iii) $\exists t<0, s<0 \in \Lambda_{B}(r)$, such that $|s|=|t|+2 m>|t|$ and $Q=\sqrt{-1} q^{-m}$.

Corollary 4.14. With the above notation, for each $t>0$, there is a non-trivial homomorphism : $W_{t}(r) \longrightarrow W_{-t}(r)$ if and only if $W_{t}(r) \cong W_{-t}(r)$. Moreover this condition is satisfied if and only if $Q=\sqrt{-1}$.

Proof. It follows from Theorem 4.13 that there is a homomorphism : $W_{t}(r) \longrightarrow$ $W_{-t}(r)$ if and only if there is a non-trivial homomorphism of $T_{r}^{a}(q)$-modules : $W_{t, z_{t}}(r) \longrightarrow W_{t, z_{-t}^{-1}}(r)$. But this happens if and only if $W_{t, z_{t}}(r) \cong W_{t, z_{-t}^{-1}}(r)$, whence the first statement.

Moreover, again by the above statement, $W_{t}(r) \cong W_{-t}(r)$ if and only if $z_{t}=z_{-t}^{-1}$. Using the value of $z_{t}$ given in Theorem 4.13 (2), one sees easily that this happens if and only if $Q^{2}=-1$.

4.5.2. Semisimplicity of endomorphism algebras. Our final result uses the cellular structure to give a precise criterion for the semisimplicity of the endomorphism algebra $\operatorname{End}_{\mathrm{U}_{q}\left(\mathfrak{s l}_{2}\right)}\left(M(\ell) \otimes V(1)^{\otimes r}\right)$, which may also be deduced from results in [5].

Theorem 4.15. Assume that $q$ is not a root of unity in $\mathcal{K}_{0}$. The endomorphism algebra $\operatorname{End}_{\mathrm{U}_{q}\left(\mathfrak{s l}_{2}\right)}\left(M(\ell) \otimes V(1)^{\otimes r}\right)$ is non-semisimple for all $r$ if $\ell=-1$. For $\ell \geqslant 0$, it is semisimple if and only if $r \leqslant \ell+1$.

Proof. When $\ell=-1, Q=\sqrt{-1}$. Hence by Corollary 4.14, there are coincidences among the cell modules of $\mathbb{\mathbb { L }} \mathbb{B}_{r}(q, Q)$, whence the endomorphism algebra is neither semisimple nor quasi-hereditary. This proves the first statement.

Now assume $\ell>-1$. We apply the criteria in (4.27) in the case where $Q=$ $\sqrt{-1} q^{-(\ell+1)}$. For the criterion (i) to apply, we require $t+m=\ell+1>0$; (ii) cannot apply in any case, while for (iii) we require $m=\ell+1>0$.

Now in case (i) we require $t+2 m=\ell+1+m \leqslant r$ for some $m>0$, so $r \geqslant \ell+2$. In case (iii), we require $m=\ell+1$ and $|t|+2 m \leqslant r$, i.e. $r>2(\ell+1) \geqslant \ell+3$. This shows that $\operatorname{End}_{\mathrm{U}_{q}\left(\mathfrak{s l}_{2}\right)}\left(M(\ell) \otimes V(1)^{\otimes r}\right)$ is semisimple for $r \geqslant \ell+1$.

Consider now the case $r=\ell+2$ (where $\ell \geqslant 0$ ). We show that there is always a non-trivial homomorphism : $W_{\ell+2}(\ell+2) \longrightarrow W_{(\ell)}(\ell+2)$. For this, observe that in the notation above, $z_{\ell}=(-1)^{\ell+\frac{3}{2}} q^{\frac{\ell}{2}+1} \sqrt{-1}$ and $z_{\ell+2}=(-1)^{\ell+\frac{3}{2}} q^{\frac{\ell}{2}+1} \sqrt{-1}=q^{-1} z_{\ell}$. Now take $t=\ell, m=1$ and $\varepsilon=1$ in (4.26). One concludes that $\left(\ell, z_{\ell}\right)<\left(\ell+2, z_{\ell+2}\right)$. This proves that $\operatorname{End}_{\mathrm{U}_{q}\left(\mathfrak{s l}_{2}\right)}\left(M(\ell) \otimes V(1)^{\otimes r}\right)$ is not semisimple for $r \geqslant \ell+2$ and $r \equiv \ell(\bmod 2)$. 
A similar argument applies for $r \geqslant \ell+2$ with $r \equiv \ell+1(\bmod 2)$, and the proof is complete.

4.6. Remarks on categories related to $\mathcal{T} \mathcal{L B}(q, \Omega)$. Note that our functor $\mathcal{F}$ is defined on the multipolar category $\mathcal{M T} \mathcal{L}(q)$. It would be interesting to determine other cases, possibly when even one restricts to the affine subcategory $\mathcal{T} \mathcal{L}^{a}(q)$, where one still has an equivalence. We mention finally that endomorphisms of $\mathcal{M T} \mathcal{L}(q)$ and $\mathcal{M T} \mathcal{L}(q, \Omega)$ in the multipolar case give rise to algebras closely related to the affine Temperley-Lieb algebra, e.g., $\operatorname{End}_{\mathcal{M T} \mathcal{L}(q)}\left(m, v^{r}, m\right)$ and $\operatorname{End}_{\mathcal{M T L}(q, \Omega)}\left(m, v^{r}, m\right)$. The functor $\mathcal{F}$ enables us to investigate the representation theory of such algebras via the quantum group $\mathrm{U}_{q}\left(\mathfrak{s l}_{2}\right)$.

\section{ACKNOWLEDGMENT}

The authors thank the Research Institute at Oberwolfach for its hospitality at an RIP program, where this work began.

\section{REFERENCES}

[1] Daniel Allcock, Braid pictures for Artin groups, Trans. Amer. Math. Soc. 354 (2002), no. 9, 3455-3474, DOI 10.1090/S0002-9947-02-02944-6. MR.1911508

[2] Henning H. Andersen, Gustav I. Lehrer, and Ruibin Zhang, Cellularity of certain quantum endomorphism algebras, Pacific J. Math. 279 (2015), no. 1-2, 11-35, DOI 10.2140/pjm.2015.279.11. MR3437768

[3] Tomoyuki Arakawa and Takeshi Suzuki, Duality between $\mathfrak{s l}_{n}(\mathbf{C})$ and the degenerate affine Hecke algebra, J. Algebra 209 (1998), no. 1, 288-304, DOI 10.1006/jabr.1998.7530. MR 1652134

[4] Joseph Bernstein, Igor Frenkel, and Mikhail Khovanov, A categorification of the TemperleyLieb algebra and Schur quotients of $U\left(\mathfrak{s l}_{2}\right)$ via projective and Zuckerman functors, Selecta Math. (N.S.) 5 (1999), no. 2, 199-241, DOI 10.1007/s000290050047. MR.1714141

[5] Jonathan Brundan and Catharina Stroppel, Highest weight categories arising from Khovanov's diagram algebra III: category $\mathcal{O}$, Represent. Theory 15 (2011), 170-243, DOI 10.1090/S1088-4165-2011-00389-7. MR2781018

[6] Vyjayanthi Chari and Andrew Pressley, Quantum affine algebras and affine Hecke algebras, Pacific J. Math. 174 (1996), no. 2, 295-326. MR1405590

[7] Anton Cox, John Graham, and Paul Martin, The blob algebra in positive characteristic, J. Algebra 266 (2003), no. 2, 584-635, DOI 10.1016/S0021-8693(03)00260-6. MR 1995129

[8] Zajj Daugherty, Arun Ram, and Rahbar Virk, Affine and degenerate affine BMW algebras: the center, Osaka J. Math. 51 (2014), no. 1, 257-283. MR3192543

[9] V. G. Drinfel'd, Degenerate affine Hecke algebras and Yangians (Russian), Funktsional. Anal. i Prilozhen. 20 (1986), no. 1, 69-70. MR831053

[10] Igor Frenkel, Mikhail Khovanov, and Catharina Stroppel, A categorification of finitedimensional irreducible representations of quantum $\mathfrak{s l}_{2}$ and their tensor products, Selecta Math. (N.S.) 12 (2006), no. 3-4, 379-431, DOI 10.1007/s00029-007-0031-y. MR2305608

[11] Peter J. Freyd and David N. Yetter, Braided compact closed categories with applications to low-dimensional topology, Adv. Math. 77 (1989), no. 2, 156-182, DOI 10.1016/00018708(89)90018-2. MR.1020583

[12] J. J. Graham and G. I. Lehrer, Cellular algebras, Invent. Math. 123 (1996), no. 1, 1-34, DOI 10.1007/BF01232365. MR1376244

[13] J. J. Graham and G. I. Lehrer, The representation theory of affine Temperley-Lieb algebras, Enseign. Math. (2) 44 (1998), no. 3-4, 173-218. MR.1659204

[14] J. J. Graham and G. I. Lehrer, Diagram algebras, Hecke algebras and decomposition numbers at roots of unity (English, with English and French summaries), Ann. Sci. École Norm. Sup. (4) 36 (2003), no. 4, 479-524, DOI 10.1016/S0012-9593(03)00020-X. MR2013924

[15] K. Iohara, G. I. Lehrer, and R. B. Zhang, Temperley-Lieb algebras at roots of unity, a fusion category and the Jones quotient, Math. Res. Lett. 26 (2019), no. 1, 121-158, DOI 10.4310/MRL.2019.v26.n1.a8. MR3963979 
[16] Michio Jimbo, A q-analogue of $U(\mathfrak{g l}(N+1))$, Hecke algebra, and the Yang-Baxter equation, Lett. Math. Phys. 11 (1986), no. 3, 247-252, DOI 10.1007/BF00400222. MR841713

[17] V. F. R. Jones, Hecke algebra representations of braid groups and link polynomials, Ann. of Math. (2) 126 (1987), no. 2, 335-388, DOI 10.2307/1971403. MR908150

[18] G. I. Lehrer and R. B. Zhang, Strongly multiplicity free modules for Lie algebras and quantum groups, J. Algebra 306 (2006), no. 1, 138-174, DOI 10.1016/j.jalgebra.2006.03.043. $\operatorname{MR} 2271576$

[19] G. I. Lehrer and R. B. Zhang, A Temperley-Lieb analogue for the BMW algebra, Representation theory of algebraic groups and quantum groups, Progr. Math., vol. 284, Birkhäuser/Springer, New York, 2010, pp. 155-190, DOI 10.1007/978-0-8176-4697-4_7. MR2761939

[20] G. I. Lehrer and R. B. Zhang, The Brauer category and invariant theory, J. Eur. Math. Soc. (JEMS) 17 (2015), no. 9, 2311-2351, DOI 10.4171/JEMS/558. MR3420509

[21] Tobias Lejczyk and Catharina Stroppel, $A$ graphical description of $\left(D_{n}, A_{n-1}\right)$ Kazhdan-Lusztig polynomials, Glasg. Math. J. 55 (2013), no. 2, 313-340, DOI 10.1017/S0017089512000547. MR3040865

[22] George Lusztig, Affine Hecke algebras and their graded version, J. Amer. Math. Soc. 2 (1989), no. 3, 599-635, DOI 10.2307/1990945. MR991016

[23] Paul Martin and Hubert Saleur, The blob algebra and the periodic Temperley-Lieb algebra, Lett. Math. Phys. 30 (1994), no. 3, 189-206, DOI 10.1007/BF00805852. MR1267001

[24] N. Yu. Reshetikhin and V. G. Turaev, Ribbon graphs and their invariants derived from quantum groups, Comm. Math. Phys. 127 (1990), no. 1, 1-26. MR.1036112

[25] N. Reshetikhin and V. G. Turaev, Invariants of 3-manifolds via link polynomials and quantum groups, Invent. Math. 103 (1991), no. 3, 547-597, DOI 10.1007/BF01239527. MR1091619

[26] J. D. Rogawski, On modules over the Hecke algebra of a p-adic group, Invent. Math. 79 (1985), no. 3, 443-465, DOI 10.1007/BF01388516. MR782228

[27] V. G. Turaev, Quantum invariants of knots and 3-manifolds, De Gruyter Studies in Mathematics, vol. 18, Walter de Gruyter \& Co., Berlin, 1994. MR1292673

[28] A. V. Zelevinsky, Induced representations of reductive $\mathfrak{p}$-adic groups. II. On irreducible representations of $\mathrm{GL}(n)$, Ann. Sci. École Norm. Sup. (4) 13 (1980), no. 2, 165-210. MR584084

[29] R. B. Zhang, M. D. Gould, and A. J. Bracken, Quantum group invariants and link polynomials, Comm. Math. Phys. 137 (1991), no. 1, 13-27. MR1099254

Univ Lyon, Université Claude Bernard Lyon 1, CNRS UMR 5208, Institut Camille Jordan, 43 Boulevard du 11 Novembre 1918, F-69622 Villeurbanne Cedex, France

Email address: iohara@math.univ-lyon1.fr

School of Mathematics and Statistics, University of Sydney, N.S.W. 2006, Australia Email address: gustav.lehrer@sydney.edu.au

School of Mathematics and Statistics, University of Sydney, N.S.W. 2006, Australia Email address: ruibin.zhang@sydney.edu.au 